See discussions, stats, and author profiles for this publication at: https://www.researchgate.net/publication/325786472

\title{
Optimal portfolio choice with benchmarks
}

Article in Journal of the Operational Research Society · June 2018

DOI: 10.1080/01605682.2018.1470066

CITATIONS

3 authors:

Carole Bernard

University of Waterloo

95 PUBLICATIONS 990 CITATIONS

SEE PROFILE

2. Steven Vanduffel

19. Vrije Universiteit Brussel

125 PUBLICATIONS 1,356 CITATIONS

SEE PROFILE

Some of the authors of this publication are also working on these related projects:

Project Variable Annuities Modeling View project

Project Optimal Portfolios View project
READS

36

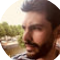

Rob Hendrik De Staelen

Ghent University

37 PUBliCATIONS 91 CITATIONS

SEE PROFILE 


\title{
Optimal Portfolio Choice with Benchmarks
}

\author{
Carole Bernard, Rob H. De Staelen’ Steven Vanduffel ${ }^{\ddagger}$
}

March 15, 2018

\begin{abstract}
We construct an algorithm that makes it possible to numerically obtain an investor's optimal portfolio under general preferences. In particular, the objective function and risks constraints may be driven by benchmarks (reflecting state-dependent preferences). We apply the algorithm to various classic optimal portfolio problems for which explicit solutions are available and show that our numerical solutions are compatible with them. This observation allows us to conclude that the algorithm can be trusted as a viable way to deal with portfolio optimization problems for which explicit solutions are not in reach.
\end{abstract}

Key-words: optimal portfolio, algorithm, law-invariant, GOP, cost-efficiency, state-dependent preferences.

\section{Introduction}

The quest for truly quantitative approaches to optimal portfolios was effectively initiated by Markowitz (1952). It is known that his mean-variance optimization framework, which balances return and risk (measured by mean and variance, respectively), provides the same solutions as those obtained when maximizing the expected utility of the portfolio using a quadratic utility function (Expected Utility Theory (EUT)). Although it is not reasonable to expect investors have all to adhere to using quadratic utility, Levy \& Markowitz (1979), Markowitz (1952) and Markowitz et al. (2000) have all asserted that the theoretical

\footnotetext{
${ }^{*}$ C. Bernard, Grenoble Ecole de Management, Department of Accounting, Law and Finance, 12 rue Pierre Sémard, 38000 Grenoble, France. Vrije Universiteit Brussel, Department of Economics and Political Science, Belgium (e-mail: carole.bernardegrenoble-em.com)

${ }^{\dagger}$ R. H. De Staelen, Ghent University, Department of Mathematical Analysis, Sint-Pietersnieuwstraat 41, 9000 Gent, Belgium. (e-mail: rob. destaelen@ugent . be)

$\ddagger$ S. Vanduffel, Vrije Universiteit Brussel (VUB), Department of Economics and Political Science, Pleinlaan 2, 1050 Brussel, Belgium. (e-mail: steven.vanduffelevub.ac.be)
} 
gap between expected utility maximization and mean-variance analysis is not significant in practice.

Nonetheless, studies by Allais (1953), Edwards (1955, 1962) and Machina (1987, 1995. 2004) have contributed to a growing body of evidence that individuals do not necessarily conform to the key assumptions or predictions of expected utility models. This has led to the development of alternative decision theories that seek to accommodate systematic departures from the expected utility model while retaining as much of its analytical power as possible. Other paradigms that have been proposed in the literature include Yaari's Dual Theory (Yaari 1987), Lopes' SP / A Theory (Lopes 1987, Lopes \& Oden 1999, Shefrin \& Statman 2000), Quiggin's Rank Dependent Utility Theory (Quiggin 1993) and the Cumulative Prospect Theory of Kahneman \& Tversky (Tversky \& Kahneman 1992). Although these competing theories differ significantly from each other, they have a common ground in that the wealth level is evaluated using an increasing law-invariant objective 11 Specifically, the investor does not care about the particular states of the economy in which the outcomes of a payoff are received; he or she cares only about the distribution of this payoff.

Dybvig (1988) introduced an alternative method for portfolio selection. Rather than optimizing an increasing law-invariant objective for a given budget (primal problem), $\mathrm{Dy}-$ bvig minimized the budget necessary to obtain a desired distribution of terminal wealth (dual problem). He showed that in order to generate a given distribution at minimum cost, the payoff must be decreasing in the state-price process; see also Bernard et al. (2014a) and Carlier \& Dana (2011). It is thus optimal for investors to consume more in cheaper states, which reflect economic abundance, than in the more expensive ones, which reflect economic recession. Clearly, solutions to the primal problem must also have this property of monotonicity. In this paper, we exploit this monotonicity property to propose an efficient method that makes it possible to numerically obtain optimal portfolios in law-invariant frameworks. This result is useful, as analytical solutions $2^{2}$ are often not in reach and typically come at the cost of over-simplifying assumptions.

One problem, however, with optimal portfolios derived in law-invariant frameworks

\footnotetext{
${ }^{1}$ Bernard et al. (2015a) show that this is equivalent to having preferences that satisfy first-order stochastic dominance (FSD). Interestingly, many economists consider a violation of this property as grounds for refuting a particular theory; see e.g., Birnbaum (1997), Birnbaum \& Navarrette (1998), Levy (2008) for further discussions and empirical evidence of FSD violations. To illustrate the importance of FSD consistency in the literature, note for instance that Kahneman \& Tversky (1979) have developed the cumulative prospect theory (Tversky \& Kahneman (1992)) in order to address the FSD violation of their original prospect theory.

2In the literature, a significant number of papers solve portfolio problems in a rather ad-hoc fashion and aim at obtaining explicit formulae. In this regard, we refer to Merton's expected utility problem (Merton 1969. 1971), Merton's problem with the Basak-Shapiro Value-at-Risk constraint (Basak \& Shapiro 2001), Browne's target probability optimization problem (Browne 1999), the optimal portfolio problem for a loss-averse investor as in Berkelaar et al. (2004) and optimal choice under Yaari's dual theory (Yaari 1987, He \& Zhou 2011).
} 
is that they provide their best outcomes in states of economic abundance, whereas many investors would prefer to receive optimal payouts when the need is greatest, i.e., in states of economic recession. A practical illustration of such considerations is the existence of the insurance business. Specifically, most people are more inclined to receive money from an insurer in the case of an event such as a fire destroying their property than to receive the same amount as the result of a favorable lottery draw (Bernard \& Vanduffel 2014). Many fund managers also choose their portfolio composition conditionally on knowing the value of some other portfolios or market indices (benchmarks). For example, changes in portfolio weights can be driven by a volatility index such as the VIX, changes in interest rates or a more general market index (Roll 1992). Moreover, the performance of fund managers is often assessed relative to that of the sector in which they invest (Daniel et al.1997), leading to a tendency to benchmark accordingly. In all these cases, optimal investment decisions are driven not by the pursuit of a law invariant objective, but by state-dependent preferences. A possible model that encompasses all the above examples is presented in Bernard et al. (2015b), who also derive a characterization of optimal portfolios for state-dependent preferences. Specifically, they extend Dybvig's work by showing that when investors aim at optimizing an objective that depends on a benchmark, the optimal portfolio is decreasing in the state-price process, conditionally on this benchmark. In this paper, we exploit this property to outline a numerical framework that makes it possible to obtain the optimal portfolio for any state-dependent objective. We apply the algorithm to several classic portfolio problems for which explicit solutions exist and show that our numerically obtained solutions match them closely.

The algorithm that we propose makes it possible to deal efficiently with a wide range of optimal portfolio selection problems for which no explicit solutions are readily available. In particular, the algorithm allows us to incorporate in optimal portfolio selection problems all types of risk constraints needed to build a realistic model. Doing so typically implies that an explicit solution will no longer be available, but has the advantage of providing an approximate solution to a problem that otherwise would be difficult to solve explicitly. For example, we find the optimal portfolio for an investor facing a CoVaR constraint (Adrian \& Brunnermeier 2011). The CoVaR recently appeared as a risk measure to assess risk of systemically important financial institutions (SIFIs). The key insight is that an important component of the risk that a SIFI represents for the global economy (systemic risk) arises from its dependence with the global financial system; higher systemic risk should be compensated by higher capital requirements. Similarly, it appears reasonable to develop strategies that aim at generating a sufficient level of income when the financial system as 
a whole is under stress. Technically, we extend the optimization of expected utility with a VaR constraint (probability constraint) of Basak \& Shapiro (2001) to a CoVaR constraint, i.e., a conditional probability constraint, in which the conditioning event is a crisis. Finally, the algorithm that we propose to deal with optimization problems for which no explicit solutions are known may also be used to potentially guess the analytical solution of the optimization problem at hand and to prove its optimality.

The paper is organized as follows. In Section 2 we describe the financial market and the general form of the optimal portfolio selection problems that we consider. In Section 3 we specifically study law-invariant portfolio problems and provide an algorithm that makes it possible to numerically obtain the respective optimal portfolios in these cases. We apply it to some classical (non-)expected utility problems for which explicit solutions are available and show that the numerical solutions are compatible with these. In Section 4 we add state-dependent constraints to the setting and extend the algorithm to this case. We study some examples in which the explicit solution is available and show that the algorithm is able to reproduce them. We use the algorithm to solve relevant investment problems (e.g., optimal portfolio choice under a CoVaR constraint) for which no explicit solution is known. Final remarks are presented in Section 5 .

\section{Setting}

Here we present the market model and describe the general form of portfolio problems that we study in this paper.

\subsection{Market Model}

We consider an investor with a fixed horizon $T$ without intermediate consumption. We assume an arbitrage-free market in which a payoff $X_{T}$ received at time $T$ can be valued at present time as the expectation of $X_{T}$ multiplied by the state-price density ${ }^{3} \xi_{T}$, i.e., its price $c\left(X_{T}\right)$ is given as $c\left(X_{T}\right)=\mathbb{E}\left[\xi_{T} X_{T}\right]$, where the expectation is taken under the real-world probability $\mathbb{P}$. (We refer to Björk (2004) for extensive theory on arbitrage-free pricing.) In other words, $\xi_{T}$ is the discount factor that is used to compute the initial cost of $X_{T}$. Platen \& Heath (2006) show that, under some general assumptions, discounting can be done using the Growth Optimal Portfolio (GOP) as numéraire (deflator). Specifically, the GOP is a portfolio with the property that it will almost surely accumulate more wealth

\footnotetext{
${ }^{3}$ The state-price $\xi_{t}(\omega)$ is the price per unit of probability $\mathbb{P}$ of the "atomic" time and state-contingent claim (Arrow-Debreu security) that delivers one unit of a specific consumption good if a specific uncertain state $\omega$ realizes at a specific future date $t$. For more information we refer to Eeckhoudt et al. (2011).
} 
than any other strategy at an infinite horizon. It can also be seen as the portfolio that has maximum expected log-utility of terminal wealth at any horizon. Similarly to Platen \& Heath (2006), we use $S_{T}^{\star}$ as numéraire and obtain that $c\left(X_{T}\right)=\mathbb{E}\left[\frac{X_{T}}{S_{T}^{\star}}\right]$, in which $S_{T}^{\star}$ is the value at maturity $T$ of one unit invested at time $t=0$ in the GOP, i.e., $\xi_{T}=1 / S_{T}^{\star}$. Note that in the Black-Scholes market set-up the use of the GOP as numéraire for pricing payoffs is the unique approach that is consistent with no-arbitrage.

\subsection{Optimal Portfolio Choice}

The optimal portfolio choice problem that we consider throughout the paper can be generically formulated as

$$
\max _{X_{T} \in \mathcal{A}} V\left(X_{T}\right)
$$

in which $V(\cdot)$ is the objective and the admissible set $\mathcal{A}$ is of the form

$$
\mathcal{A}=\left\{\begin{array}{l|c}
X_{T} & c\left(X_{T}\right) \leqslant W_{0} \quad \text { (budget constraint) } \\
& \text { risk constraints on } X_{T}
\end{array}\right\} .
$$

A risk constraint can typically be expressed as $\rho\left(X_{T}\right) \leqslant \rho_{0}$, in which $\rho(\cdot)$ is a risk measure and $\rho_{0}$ is the maximum risk exposure. Note that the objective $V(\cdot)$ may depend solely on the distribution of the final wealth $X_{T}$ (i.e., $V$ is law-invariant), but it may also be statedependent in that the states in which cash flows are received matter. The same feature holds true for the admissible set $\mathcal{A}$ : the risk constraints may be law-invariant or statedependent. When both the objective function and the risk constraints are law-invariant, we say that the preferences are law-invariant (Section 3); otherwise, they are state-dependent (Section 4).

\section{Optimal Portfolio for Law-Invariant Preferences}

In this section, we assume law-invariant preferences. Dybvig (1988), Carlier \& Dana (2011) and Bernard et al. (2014a) show that in this case solutions to optimal portfolio problems must be decreasing in the state-price density and thus increasing in $S_{T}^{\star} 4^{4}$ This property plays a key role in designing a new discrete algorithm that makes it possible to find the optimal portfolio efficiently.

We first lay out the algorithm and next apply it to several classic optimization prob-

\footnotetext{
${ }^{4}$ Here, increasing refers to "non-decreasing". Specifically, it does not mean that the portfolio is strictly increasing in $S_{T}^{\star}$.
} 
lems for which an explicit solution has been reported in the literature, such as the Merton problem of maximizing expected utility (Merton 1969, 1971), the Merton problem of maximizing expected utility in the presence of a Value-at-Risk (VaR) constraint (Basak \& Shapiro 2001), the optimal portfolio problem for the loss-averse investor (Berkelaar et al. 2004), and the optimal portfolio problem under Yaari's dual theory (Yaari 1987, He \& Zhou 2011). These examples show that the algorithm performs admirably in reproducing the explicit solution and provides evidence that the algorithm can be used to solve any other law-invariant optimal portfolio selection problem. In the second part of the paper we extend our algorithm to accommodate state-dependent preferences.

\subsection{Optimal Portfolio Choice Algorithm}

Set-up: The algorithm that we propose first requires a discretization of the problem. Let $s_{1}^{\star}, s_{2}^{\star}, \ldots, s_{n}^{\star}$ be $n$ equiprobable (with respect to the real-world probability $\mathbb{P}$ ) ordered realizations (states) of the GOP, $s_{1}^{\star}<s_{2}^{\star}<\cdots<s_{n}^{\star}$. These realizations can be obtained by Monte Carlo simulations. In this paper, they are computed by inverting the distribution $F_{S_{T}^{\star}}$ of the GOP $S_{T}^{\star}$ as follows:

$$
s_{k}^{\star}:=F_{S_{T}^{\star}}^{-1}\left(\frac{k-0.5}{n}\right), \quad \text { for } k=1,2, \ldots, n .
$$

The discrete counterpart $5^{5}$ to Problem (1a) can be formulated as

$$
\max _{\left(x_{1}, x_{2}, \ldots, x_{n}\right) \in \mathcal{A}^{d}} f\left(x_{1}, x_{2}, \ldots, x_{n}\right)
$$

in which the admissible set $\mathcal{A}^{d}$ (counterpart to $(1 \mathrm{~b})$ ) is given as

$$
\mathcal{A}^{d}:=\left\{\left(x_{1}, x_{2}, \ldots, x_{n}\right) \in \mathbb{R}^{n} \mid \begin{array}{c}
\frac{1}{n} \sum_{i=1}^{n} \frac{x_{i}}{s_{i}^{\star}} \leqslant W_{0} \\
\text { risk constraints on }\left(x_{1}, x_{2}, \ldots, x_{n}\right)
\end{array}\right\} .
$$

For the ease of presentation we omit the additional risk constraints $\varsigma^{6}$ when describing the algorithm. Using the monotonicity property, the admissible set $(3 b)$ can be restricted to the set

$$
\left\{\begin{array}{l|c}
\left(x_{1}, x_{2}, \ldots, x_{n}\right) \in \mathbb{R}^{n} & \begin{array}{c}
x_{1} \leqslant x_{2} \leqslant \cdots \leqslant x_{n} \\
\frac{1}{n} \sum_{i=1}^{n} \frac{x_{i}}{s_{i}^{\star}} \leqslant W_{0}
\end{array}
\end{array}\right\} .
$$

\footnotetext{
${ }^{5}$ A standard example of a law-invariant objective function is the expected utility functional, in which $V(X)=\mathbb{E}[u(X)]$. For the discretized version we would then obtain that $f\left(x_{1}, x_{2}, \ldots, x_{n}\right)=\frac{1}{n} \sum_{i=1}^{n} u\left(x_{i}\right)$.

${ }^{6}$ An example of risk constraint is a Value-at-Risk constraint, i.e. $\mathbb{P}\left(X_{T} \geqslant \underline{W}\right) \geqslant 1-\alpha$, so that assuming $x_{1} \leqslant x_{2} \leqslant \cdots \leqslant x_{n}$, and $\frac{k}{n}=\alpha$, it amounts to constraining $\left(x_{1}, x_{2}, \ldots, x_{n}\right)$ in that $\underline{W} \leqslant x_{k}$ must hold.
} 
Using the auxiliary variables $y_{i}=x_{i}-x_{i-1}$ (with the convention $x_{0}=0$ ), $i=1,2, \ldots, n$, we can then rewrite the optimization problem as

$$
\max _{\left(y_{1}, y_{2}, \ldots, y_{n}\right) \in \mathcal{B}} f\left(y_{1}, y_{1}+y_{2}, \ldots, y_{1}+y_{2}+\cdots+y_{n}\right)
$$

in which the admissible set is now given as

$$
\mathcal{B}:=\left\{\left(y_{1}, y_{2}, \ldots, y_{n}\right) \in\left(\mathbb{R}_{+}\right)^{n} \mid \sum_{j=1}^{n} \zeta_{j} y_{j} \leqslant W_{0}\right\}, \quad \zeta_{j}:=\frac{1}{n} \sum_{k=j}^{n} \frac{1}{s_{k}^{\star}} .
$$

It is clear that this discretization yields an optimization problem with a set of linear constraints. Most importantly, by rewriting the side constraint (3b) as the side constraint (4b), we greatly reduce the dimensionality of the problem, as we only need to look for solutions in the first orthant.

As the objective in $4 \mathrm{a}$ ) is generally non-concave, multiple optima can exist. However, the global optimum is known to be increasing in the GOP and our formulation (4) enforces this feature, hence avoiding the pitfall that the routine will produce a solution that cannot be a global optimum. For an overview of the theory and numerical techniques for optimization models involving one or more constraints on distribution functions we refer to Dentcheva (2006). For an in-depth review of shape constrained optimization we refer to d'Aspremont (2004). In this paper, we will rely on a built-in feature of a software package to find a minimum of a constrained nonlinear multi-variable function. Specifically, the numerical solution of (4) is constructed in MATLAB ${ }^{\circledR}(2013)$ with the help of the built-in function ${ }^{7}$ fmincon. Our routine starts with an initial guess regarding the optimum: We know that the solution must be increasing in the GOP and that its cost must be below the budget $W_{0}$. Specifically, we take as initial solution $X_{0, T}^{(0)}:=\delta \log \left(1+S_{T}^{\star}\right)$, where $\delta$ is chosen to satisfy the budget constraint and its realizations are computed in a straightforward way from those of $S_{T}^{\star}$ (see (2)). We also tested other choices for the initial guess and found that the algorithm is robust to changes in this initial condition. We have carefully designed a procedure, which consists of increasing the number of discretization points incrementally with each iteration step. It allows us to find the true solution whenever the initial discretization level $n_{0}$ (used for discretizing the initial guess $X_{0, T}^{(0)}$ ) is small enough (say, $n_{0} \leqslant 20$ ); see also the numerical examples in Sections 3 and 4 for additional evidence.

\footnotetext{
${ }^{7}$ The function implements an active-set algorithm that solves the Karush-Kuhn-Tucker (KKT) equations; see Nocedal \& Wright (2006) and Floudas \& Pardalos (2009). When the objective and constraints are twice differentiable and have Lipschitz continuous second derivatives in a neighbourhood of the optimum, the algorithm converges to the optimum when one starts close enough to it; see Chapter 18 of Nocedal \& Wright (2006) and Hanson (1981. 1999).
} 
Indeed, the number $n_{k}$ of discretization points in each iteration $(k=0,1, . ., K)$ plays an important role and has to be considered carefully. On the one hand, the larger $n_{k}$, the more unknown variables there are and the more difficult it is a priori for MATLAB ${ }^{\circledR}$ to find the optimal solution. On the other hand, the larger $n_{k}$, the better the discretization and thus the accuracy of the approximation of continuous distributions by discrete distributions. Taking into account these observations and trade-offs, we propose the following approach that refines the discretization at each step by doubling the number of discretization points whilst making it possible to improve upon the initial guess.

Description of the algorithm: First, from the initial guess $X_{0, T}^{(0)}$, obtained with very few discretization points (say, $n_{0}=20$ ) we obtain, through optimization, a rough estimate $X_{0, T}^{*}:=X_{0, T}^{\left(N_{0}\right)}$ of the optimal solution $X_{T}^{\star}$; the procedure finds iterates $X_{0, T}^{(1)}, X_{0, T}^{(2)}, X_{0, T}^{(3)}, \ldots$ and will halt when internal tolerances are met, say at $N_{0}$. The obtained estimate $X_{0, T}^{*}:=$ $X_{0, T}^{\left(N_{0}\right)}$ suffers from a too coarse discretization (as $\left.n_{0}=20\right)$, but it already has the right "shape" and has, typically, already improved the objective function significantly. Next, we double the discretization points to $n_{1}=40$ and use standard linear interpolation and extrapolation (implemented in MATLAB ${ }^{\circledR}$ ) to extend the estimate $X_{0, T}^{*}$ (20 outcomes that are increasing in a coarsely discretized GOP) to $X_{1, T}^{(0)}$ (40 outcomes related to a more finely discretized GOP), which will then serve as the new starting point of the routine. The iterations will halt, say at $N_{1}$, and a new estimate $X_{1, T}^{*}:=X_{1, T}^{\left(N_{1}\right)}$ of the optimal solution $X_{T}^{\star}$ is obtained. After repeating these steps $K$ times we have obtained $K+1$ approximations $X_{k, T}^{*}(k=$ $0, \ldots, K)$ of $X_{T}^{\star}$, each related to a more finely discretized GOP of $n_{k}=2^{k} n_{0}$ equiprobable outcomes. Algorithm PA-SIP outlines our routine in pseudo-code, which is also visualized in Figure 1 .

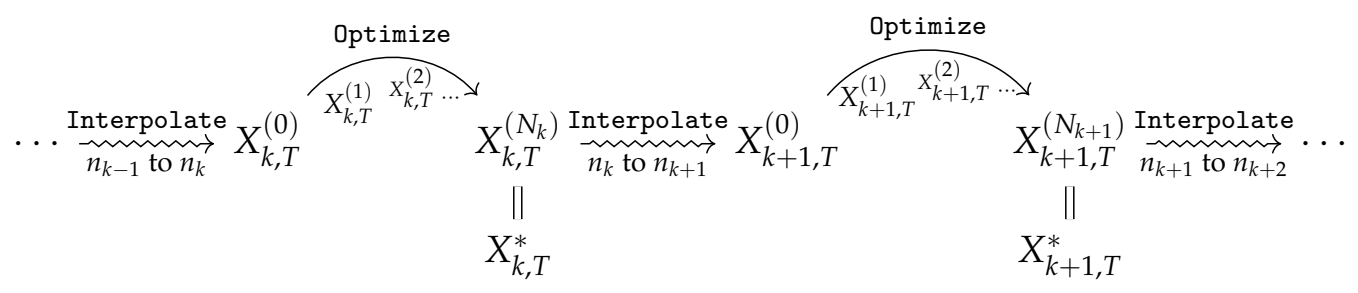

Figure 1: Diagram of Algorithm PA-SIP.

Conceiving the routine in this way makes it possible for the algorithm to find the optimal solution; by doubling the number of points five times and by using 20 points $\left(n_{0}=20\right)$ for the first run, we found in our examples that we can accurately solve optimization problems with $n_{5}=640$ discretization points, i.e., 640 unknowns to determine. 


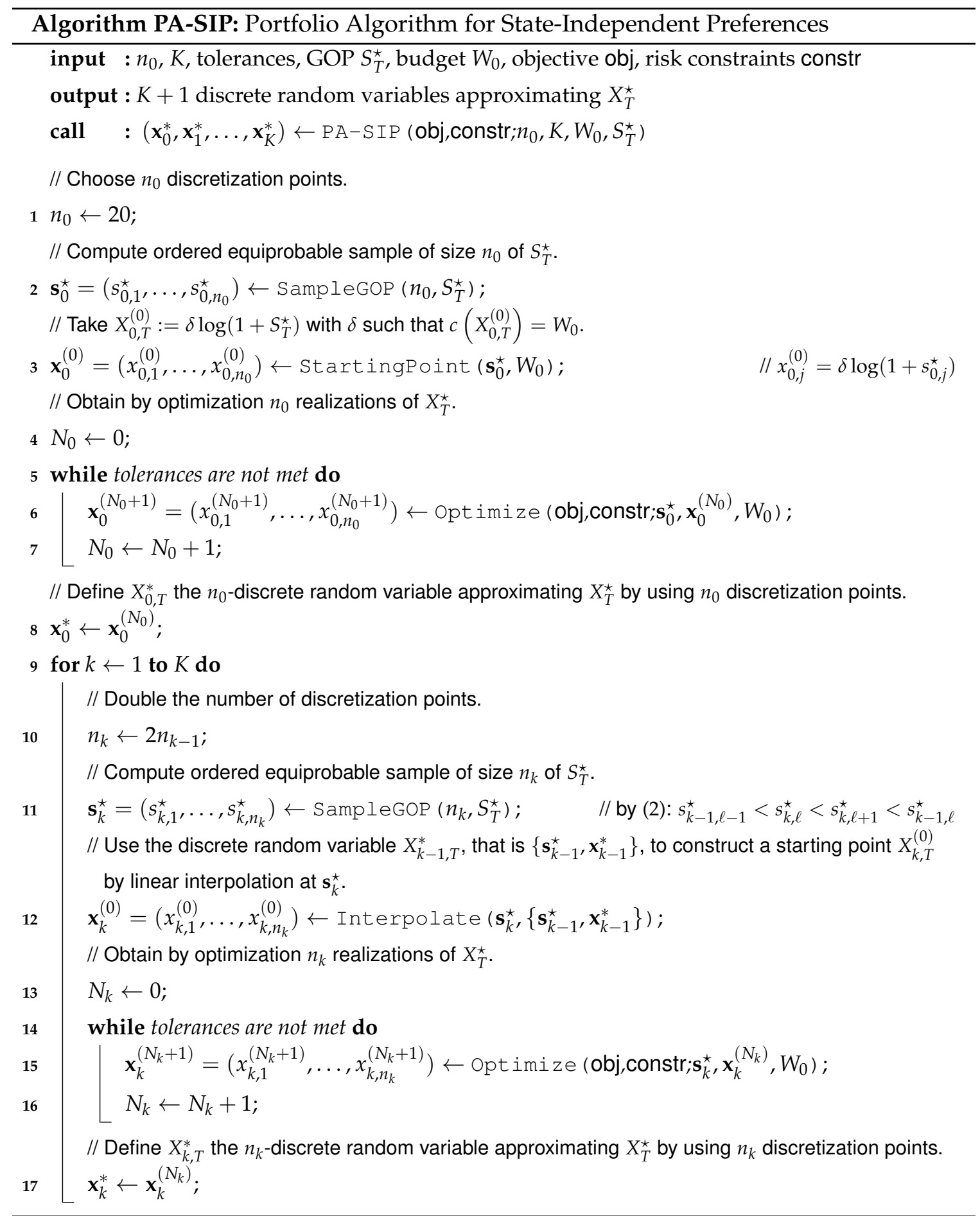

In summary, the algorithm builds crucially on the monotonicity property that the optimal solution must have with the GOP (to reduce the dimensionality of the problem) and on a careful design of the initial guess by choosing the number of discretization points dynamically. Otherwise, it would be merely a matter of luck to find a solution that is close to the true optimal one. Indeed, although the algorithm in MATLAB ${ }^{\circledR}$ is very powerful for solving multidimensional optimization problems, it will typically fail to find the global 
optimum if we run the algorithm directly with a very large number of discretization points.

In the following subsections, we apply our method to some standard optimal portfolio choice problems. Let us insist here that the goodness of the results is derived from the construction of the algorithm itself. Any naive approach consisting of directly discretizing finely a grid without a good initial condition will lead to inaccurate results and suboptimal portfolio choices.

\subsection{Application of the Portfolio Algorithm to State-Independent Preferences}

We illustrate Algorithm PA-SIP with four examples in a two-dimensional Black-Scholes market, in which individual volatility coefficients for the two assets are $\sigma_{1}$ and $\sigma_{2}$ and their correlation coefficient is $\rho_{12}$. Unless otherwise stated, the parameters are set as follows. The drift and volatility of each asset are given by $\mu_{1}=0.03, \sigma_{1}=0.20$ and $\mu_{2}=0.04, \sigma_{2}=0.30$. The risk free-rate $r$ is equal to 0.01 ; thus, the market price of risk is such that $\lambda_{\bullet}:=\frac{\mu_{1}-r}{\sigma_{1}}=$ $\frac{\mu_{2}-r}{\sigma_{2}}$ (see e.g. Bernard et al. (2011) or Bernard et al. (2015b) for a detailed description of the multidimensional Black-Scholes model). Finally, the correlation $\rho_{12}$ is taken to be equal to 0.25 , the investment horizon is $T=6$ months and the initial budget $W_{0}$ is equal to 100 . These parameters are summarized in Table 1. The GOP can then be expressed (see also

Table 1: Values of the parameters for the numerical examples.

\begin{tabular}{ccccccccc}
\hline$r$ & $\mu_{1}$ & $\mu_{2}$ & $\sigma_{1}$ & $\sigma_{2}$ & $\rho_{12}$ & $T$ & $W_{0}$ & $S_{0}^{\star}$ \\
\hline 0.01 & 0.03 & 0.04 & 0.2 & 0.3 & 0.25 & 0.5 & 100 & 1 \\
\hline
\end{tabular}

Bernard et al. (2014b)) as $S_{T}^{\star}=S_{0}^{\star} \exp \left[\left(r+\frac{\lambda^{2}}{2}\right) T+\lambda W_{T}^{\mathbb{P}}\right]$ where $\lambda=\sqrt{\frac{2}{1+\rho_{12}}} \lambda \bullet$ and $W_{T}^{\mathbb{P}}$ is a standard Brownian motion under the measure $\mathbb{P}$ at time $T$. This enables us to compute an increasingly ordered equiprobable sample of size $n$ of the GOP as

$$
s_{i}^{\star}:=\exp \left[\lambda \sqrt{T} \Phi^{-1}\left(\frac{i-0.5}{n}\right)+\left(r+\frac{\lambda^{2}}{2}\right) T\right], \quad i=1,2, \ldots, n,
$$

where $\Phi$ is the distribution function of a standard normal random variable; see also (2).

\subsubsection{Merton's expected utility problem (Merton 1971)}

A dominant decision theory in economics is the expected utility theory (EUT) of von Neumann \& Morgenstern (1947). The optimal portfolio in an EUT framework was first derived by Merton (1969, 1971), and the problem in this case is often referred to as Merton's prob- 
lem. Merton performed his analysis under Inada's conditions (Inada 1963) on $u(x)$. We recall here the analytical solution.

Proposition 3.1. Assuming that the utility function is concave, increasing and differentiable on $\mathbb{R}^{+}$, with $\lim _{x \rightarrow 0} u^{\prime}(x)=+\infty$ and $\lim _{x \rightarrow+\infty} u^{\prime}(x)=0$, the optimal payoff of the Merton expected utility problem $\max _{c\left(X_{T}\right) \leqslant W_{0}, X_{T} \geqslant 0} \mathbb{E}\left[u\left(X_{T}\right)\right]$ is given by $X_{T}^{\star}=\left[u^{\prime}\right]^{-1}\left(\frac{\theta}{S_{T}^{\star}}\right)$, where $\theta$ is determined by the budget constraint $c\left(X_{T}^{\star}\right)=W_{0}$.

Note that $X_{T}^{\star}$ in Proposition 3.1 is indeed increasing in the GOP. We illustrate our numerical method using the following utility function (CRRA utility function):

$$
u_{\eta}(w)= \begin{cases}\log (w) & \eta=1 \\ \frac{1}{1-\eta} w^{1-\eta} & \eta \neq 1\end{cases}
$$

in which $\eta>0$ is the so-called coefficient of relative risk aversion. First, we discretize the optimal portfolio choice problem according to Section 3.1. and hence consider the following discretized problem:

$$
\max _{\mathbf{y} \in \mathcal{B}} \frac{1}{n} \sum_{i=1}^{n} u_{\eta}\left(y_{1}+y_{2}+\cdots+y_{i}\right)
$$

with the admissible set as in (4b):

$$
\mathcal{B}:=\left\{\left(y_{1}, y_{2}, \ldots, y_{n}\right) \in\left(\mathbb{R}_{+}\right)^{n} \mid \sum_{j=1}^{n} \zeta_{j} y_{j} \leqslant W_{0}\right\}, \quad \zeta_{j}:=\frac{1}{n} \sum_{k=j}^{n} \frac{1}{s_{k}^{\star}} .
$$

The application of Algorithm PA-SIP leads to the results displayed in Figure 2 for $K=$ 5 , so $n_{K}=20 \cdot 2^{5}=640$.

In both panels of Figure 2, we also show the initial guess $X_{0, T}^{(0)}$ that serves as input for the algorithm, the optimal one that is obtained as its output (that is, $X_{5, T}^{*}$ ) and the analytical solution (see Proposition 3.1).

The graph presented in Panel 2(a) shows very good agreement between the theoretical and numerical results. The algorithm constructs a payoff with expected utility of 31.0256, which is exactly (as an approximation with 4 digits) the utility of the theoretical optimum. Note also that the initial guess deviates from the optimal solution, indicating that the optimization procedure is able to accommodate a suboptimal initial choice. These results are confirmed in Panel 2(b)] The numerical and theoretical solutions match closely. Moreover, our algorithm constructs a payoff with expected utility of 4.6142 , which is again very close to the exact number that is equal to 4.6141 . 


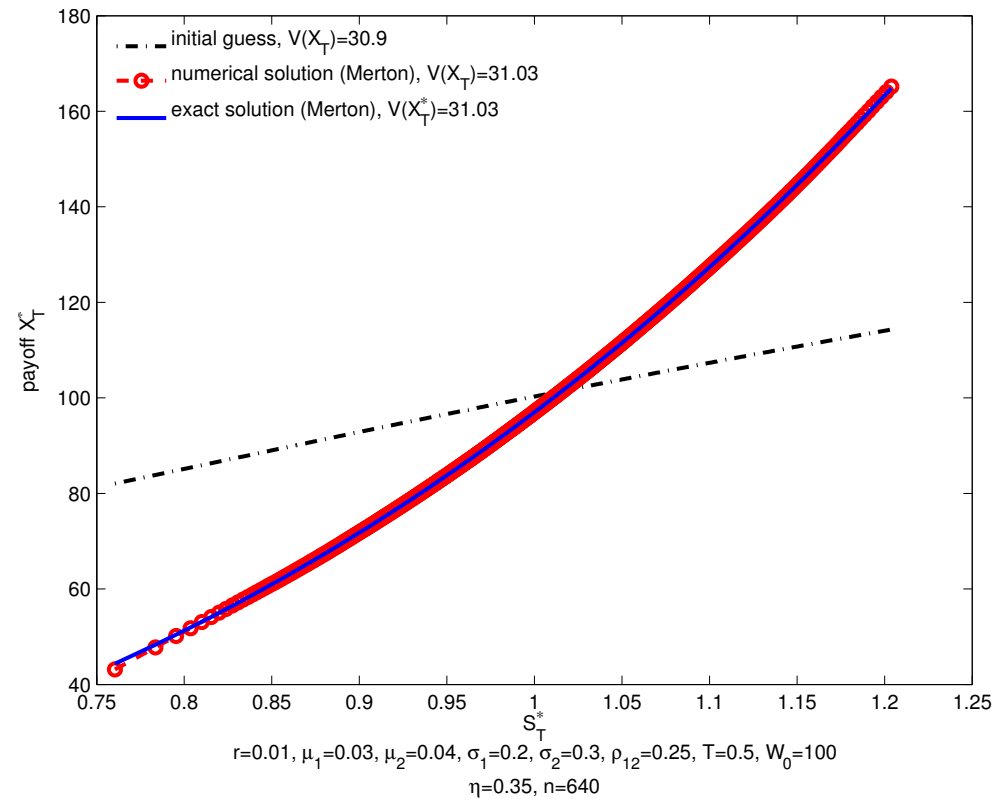

(a) $\eta=0.35$

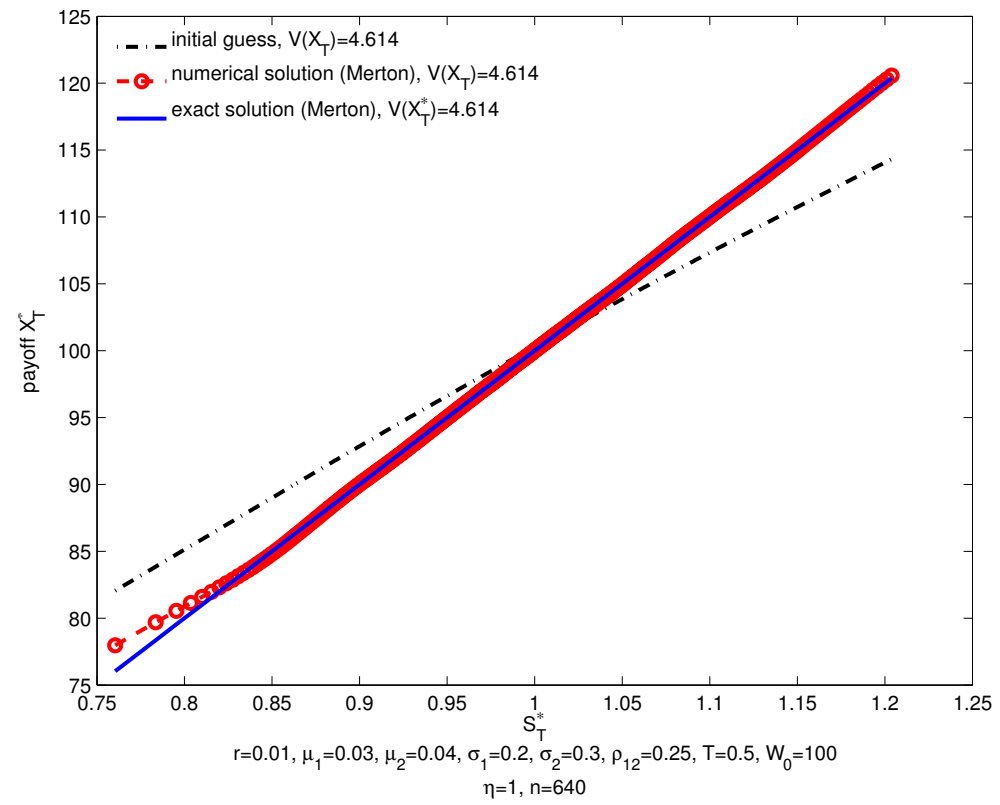

(b) $\eta=1$

Figure 2: Optimal portfolios for Merton's problem.

\subsubsection{Merton's expected utility problem with VaR constraint (Basak \& Shapiro 2001)}

The analysis of Merton's problem confirms that in a law-invariant setting the optimal payoff goes along with the GOP and thus offers little income in declining markets. Investors may feel uncomfortable with this feature. Moreover, in practice, portfolio managers often face constraints stemming from regulations and/or internal policies. Basak \& Shapiro 
(2001) incorporate these concerns into Merton's problem using a Value-at-Risk (VaR) risk measure that ensures that the minimum value of terminal wealth remains above a minimum level with desired probability.

Specifically, Basak \& Shapiro (2001) consider as a risk constraint $\rho\left(X_{T}\right):=\mathbb{P}\left(X_{T} \leqslant \underline{W}\right)$, with $\underline{W} \in \mathbb{R}^{+}$and $\rho_{0}:=\alpha \in[0,1]$; or, equivalently, they consider the risk constraint $\mathbb{P}\left(X_{T} \geqslant \underline{W}\right) \geqslant 1-\alpha$. Let us refer to

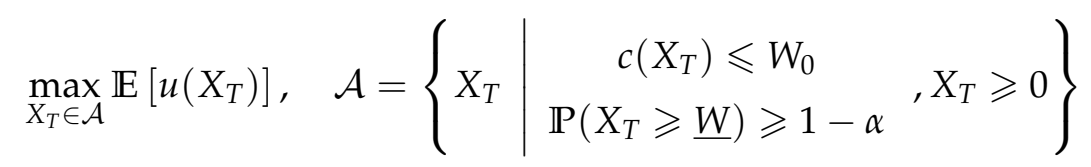

as the Basak-Shapiro VaR constrained problem. The constraint $\mathbb{P}\left(X_{T} \geqslant \underline{W}\right) \geqslant 1-\alpha$ requires an agent to have terminal wealth higher than $\underline{W}$ with probability $1-\alpha$ at least, i.e., $\operatorname{VaR}_{\alpha}\left(X_{T}\right):=F_{X_{T}}^{-1}(\alpha) \geqslant \underline{W}$. Basak \& Shapiro (2001) show that this problem can be solved explicitly and we recall their analytical solution hereafter. In this regard, note that in order to ensure that the set $\mathcal{A}$ is not empty, the budget needs to be sufficiently high, leading to the assumption $\operatorname{VaR}_{\alpha}\left(X_{T}\right):=F_{X_{T}}^{-1}(\alpha) \geqslant \underline{W}$. Finally, the Basak-Shapiro VaR constrained problem with $\alpha=0$ reduces to portfolio insurance, as the terminal wealth is constrained to be above $\underline{W}$ in all states.

Proposition 3.2. Let $0 \leqslant \underline{W} e^{-r T} \leqslant W_{0}$ and assume that the utility function $u$ is strictly increasing and that its derivative $u^{\prime}$ exists and is strictly decreasing (from $+\infty$ at 0 to $0^{+}$at $+\infty$ ). The optimal payoff $X_{T}^{\star}$ of the Basak-Shapiro VaR constrained problem is given by

$$
X_{T}^{\star}= \begin{cases}\underline{W} & S_{T}^{\star} \in\left[\underline{S}^{\star}, \bar{S}^{\star}\right), \\ {\left[u^{\prime}\right]^{-1}\left(\frac{\theta}{S_{T}^{\star}}\right)} & \text { otherwise }\end{cases}
$$

where $\theta$ is determined by the budget constraint $c\left(X_{T}^{\star}\right)=W_{0}, \bar{S}^{\star}=\theta / u^{\prime}(\underline{W})$ and $\underline{S}^{\star}$ is such that $\mathbb{P}\left(S_{T}^{\star}<\underline{S}^{\star}\right)=\alpha$.

We apply Algorithm PA-SIP to the Basak-Shapiro VaR constrained problem using the CRRA utility function $u_{\eta}$ as given in (5). We first discretize the risk constraint that appears in the admissible set: Put $k:=\lceil n \alpha\rceil \in \mathbb{N}$. Then we have

$$
\mathbb{P}\left(X_{T} \geqslant \underline{W}\right) \geqslant 1-\alpha \Longleftrightarrow F_{X_{T}}(\underline{W}) \leqslant \alpha \leqslant \frac{k}{n}=F_{X_{T}}\left(x_{k}\right) \Longrightarrow \underline{W} \leqslant x_{k}
$$

because $F_{X_{T}}\left(x_{i}\right)=F_{S_{T}^{\star}}\left(s_{i}^{\star}\right)=i / n$ (due to the monotonicity property of the optimal solution). Hence, we obtain the following discretized problem $\max _{\mathbf{y} \in \mathcal{B}} \frac{1}{n} \sum_{i=1}^{n} u_{\eta}\left(y_{1}+y_{2}+\right.$ 
$\left.\cdots+y_{i}\right)$ with the admissible set given as

$$
\mathcal{B}=\left\{\left(y_{1}, y_{2}, \ldots, y_{n}\right) \in\left(\mathbb{R}_{+}\right)^{n} \mid \begin{array}{c}
\sum_{j=1}^{n} \zeta_{j} y_{j} \leqslant W_{0} \\
y_{1}+y_{2}+\cdots+y_{\lceil n \alpha\rceil} \geqslant \underline{W}
\end{array}\right\}, \quad \zeta_{j}:=\frac{1}{n} \sum_{i=j}^{n} \frac{1}{s_{i}^{\star}} .
$$

We present the results in Figure 3 for $K=5$, so $n_{K}=20 \cdot 2^{5}=640$.

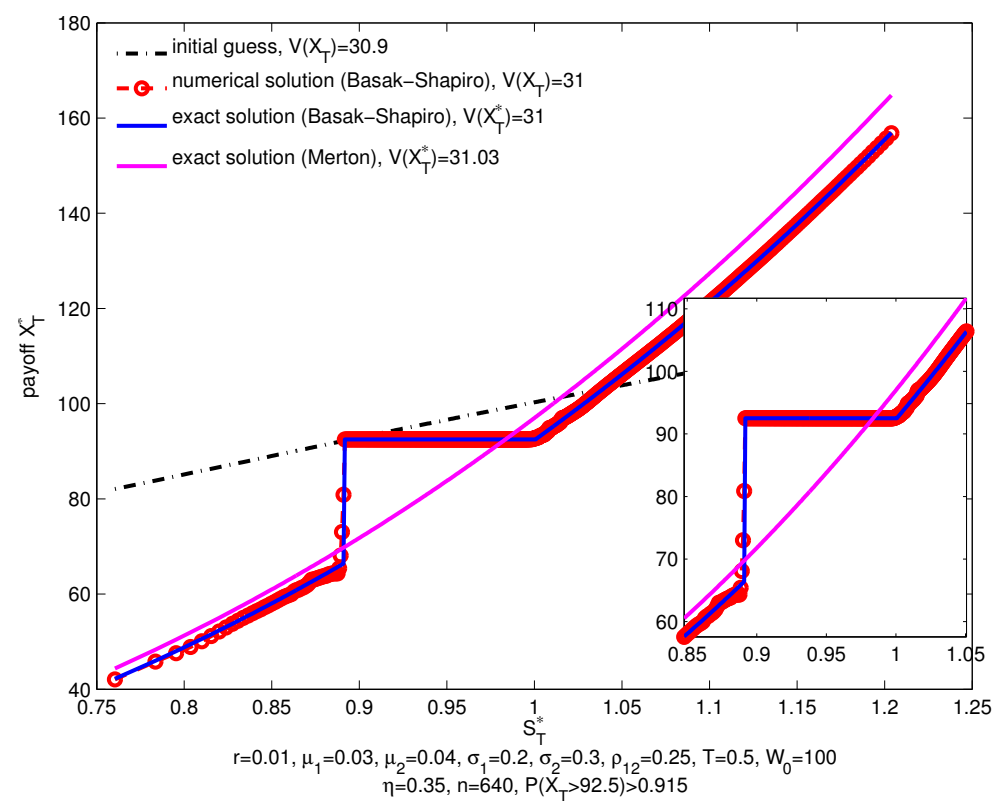

(a) $\eta=0.35$

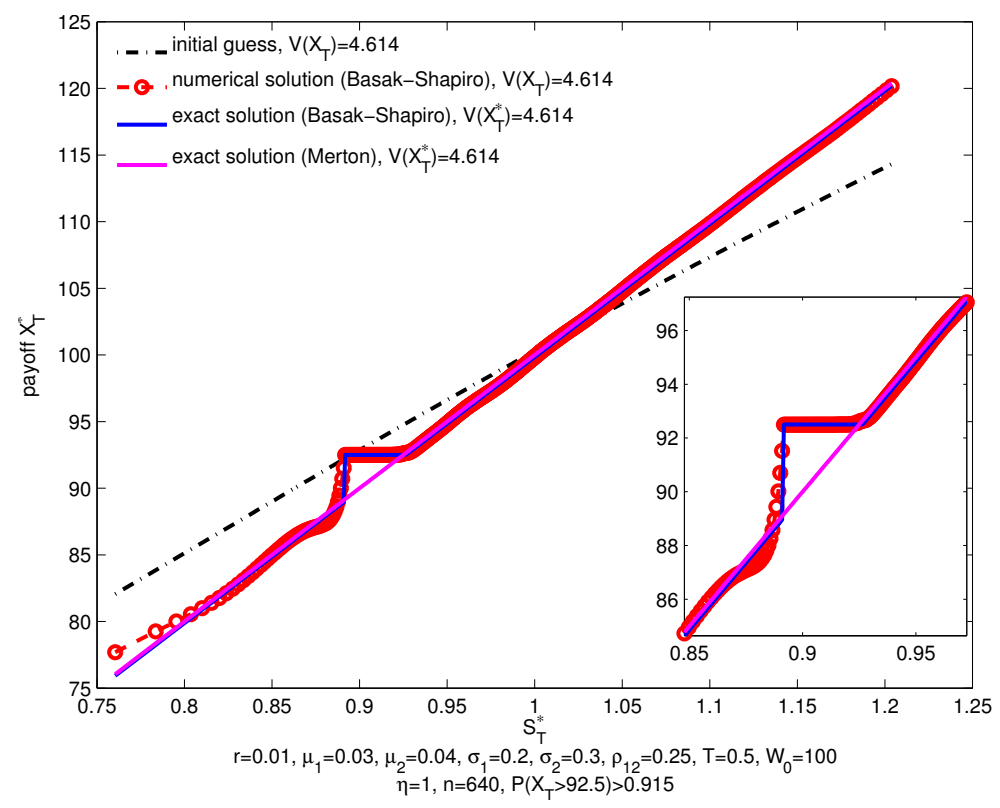

(b) $\eta=1$

Figure 3: Optimal portfolios for Merton's problem with Basak-Shapiro VaR constraint. 
In both panels of Figure 3 , we display the initial choice $X_{0, T}^{(0)}$ of the algorithm, the optimal one that is obtained as output of the algorithm (that is, $X_{5, T}^{*}$ ) and the analytical solution (Proposition 3.2).

Both panels show that there is again good agreement between the numerical and theoretical results. In particular, the algorithm is able to accurately produce the discontinuity (jump) in the analytic solution of Basak \& Shapiro (2001). Furthermore, the expected utilities of the numerical solutions closely match those of the analytical solutions.

\subsubsection{Loss-averse investor (Berkelaar et al. 2004)}

In a seminal work, Kahneman \& Tversky (1979) provided evidence that losses loom larger than gains in the perception of investors, for which reason they proposed a different utility function that is not always concave over terminal wealth. This is further developed in Berkelaar et al. (2004), who state that investors care about changes in their wealth level (with respect to a benchmark) rather than about their absolute wealth levels. In addition, the objective function is S-shaped with a convex part below the reference point and a concave part above. This setting is appealing as there is evidence that it is a realistic way in which to model preferences. Berkelaar et al. (2004) studied the optimal portfolio choice in this setting, and we refer to this problem as the optimal portfolio problem for the lossaverse investor. Specifically, one chooses the following utility function, defined over losses and gains relative to a reference level $p$ :

$$
u_{\mathrm{B}}(x)=\left\{\begin{array}{ll}
-C_{1}(p-x)^{\gamma_{1}} & x \leqslant p \\
C_{2}(x-p)^{\gamma_{2}} & p<x
\end{array} .\right.
$$

For loss aversion and increasing preferences one needs $C_{1}>C_{2}>0$. Requiring $\gamma_{i}<1$ $(i=1,2)$ ensures risk-seeking for losses (convex) and risk-aversion for gains (concave). Berkelaar et al. (2004) derive the optimal portfolio in this setting, and we recall their result first.

Proposition 3.3. The optimal payoff $X_{T}^{\star}$ of a loss-averse investor solving $\max _{c}\left(X_{T}\right) \leqslant W_{0} \mathbb{E}\left[u_{B}\left(X_{T}\right)\right]$ is given by

$$
X_{T}^{\star}=\left(p+\left(\frac{C_{2} \gamma_{2}}{\theta} S_{T}^{\star}\right)^{1 /\left(1-\gamma_{2}\right)}\right) \cdot \mathbb{1}_{\left\{\underline{S}^{\star}<S_{T}^{\star}\right\}^{\prime}}
$$


where $\underline{S}^{\star} \geqslant 0$ is the zero of the function

$$
Z(S)=\frac{1-\gamma_{2}}{\gamma_{2}}\left(\frac{S}{\theta}\right)^{\frac{\gamma_{2}}{1-\gamma_{2}}}\left(C_{2} \gamma_{2}\right)^{\frac{1}{1-\gamma_{2}}}-\frac{p \theta}{S}+C_{1} p^{\gamma_{1}}
$$

and $\theta \geqslant 0$ is determined by the budget constraint $c\left(X_{T}^{\star}\right)=W_{0}$.

Note that $X_{T}^{\star}$ in $(10)$ is indeed increasing in the GOP. Moreover, as long as the GOP stays below a threshold $\underline{S}^{\star}, X_{T}^{\star}$ takes the value zero. When the GOP is above a threshold $\underline{S}^{\star}$, the payoff $X_{T}^{\star}$ jumps to the reference level $p$ and stays above.

We illustrate the solution of the optimal portfolio problem for a maturity of $T=5$ years (so $\underline{S}^{\star}$ is clearly visible) and a reference level $p=95$. The other parameter values are $C_{1}=2.25, C_{2}=1$ and we also take $\gamma_{1}=\gamma_{2}$; see also Kahneman \& Tversky (1979) and Berkelaar et al. (2004).

The numerical results that we obtain by applying Algorithm PA-SIP are displayed in Figure 4 and contrasted with the analytical solutions. In Panel $4(\mathrm{a})$ we take $\gamma_{1}=0.88=\gamma_{2}$. Once more the numerical solution conforms well with the analytical solution. The initial guess has utility equal to 8.191 but our algorithm constructs a payoff with utility value equal to 11.005, which is again very close to the optimal value of 11.048. In Panel 4(b) we use $\gamma_{1}=0.82$ (other parameters remain unchanged). The initial guess has utility equal to 8.808. Our algorithm constructs a payoff with utility equal to 13.563, whereas the optimal value is 13.616 . 


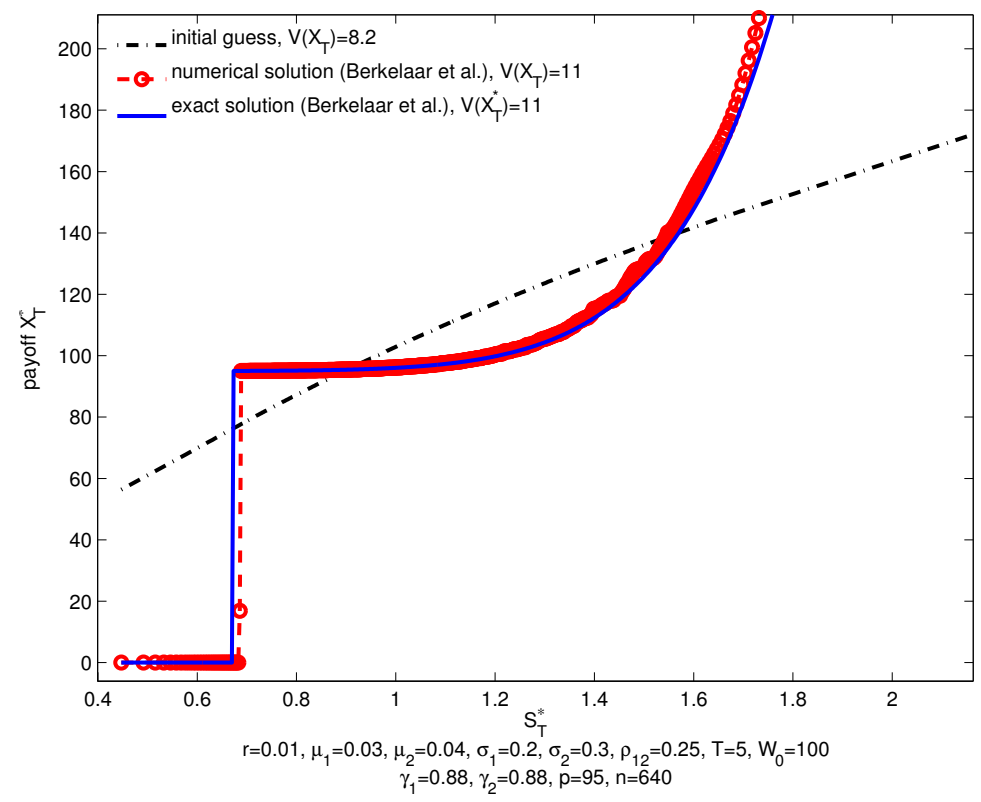

(a) $\gamma_{1}=0.88$

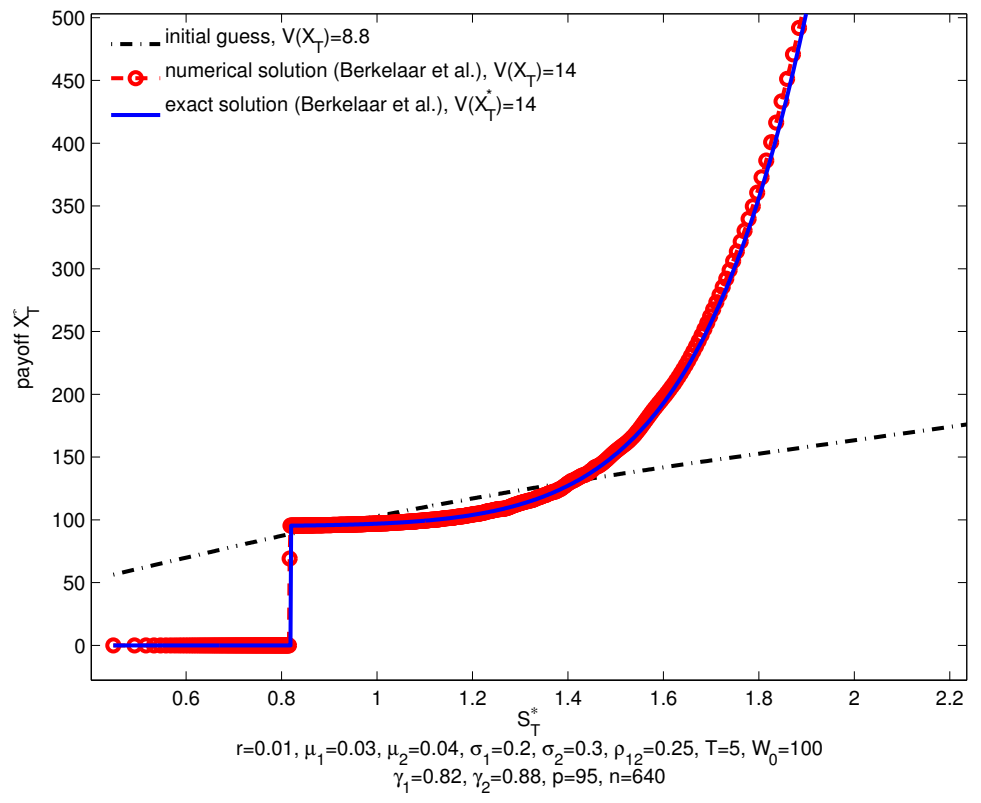

(b) $\gamma_{1}=0.82$

Figure 4: Optimal portfolios for the loss-averse investor.

\subsubsection{Optimal portfolio choice under Yaari's dual theory (Yaari 1987)}

Following Machina (1987, 1995, 2004), beginning with the work of Allais (1953) and Edwards (1955, 1962) in the early 1950s, some of the key axioms of expected utility theory have become increasingly challenged. This trend has led to the development of nonexpected utility models of risk/preferences, which seek to better reflect preferences, while 
retaining as much as possible of the analytical power of the expected utility models. The dual theory of choice of Yaari (1987) builds on a new set of axioms. In this latter framework, agents evaluate terminal wealth using the distorted expectation

$$
V\left(X_{T}\right)=\int_{0}^{+\infty} x \mathrm{~d}\left(1-w\left(1-F_{X}(x)\right)\right)=\int_{0}^{+\infty} w\left(\mathbb{P}\left[X_{T}>x\right]\right) \mathrm{d} x
$$

where $w:[0,1] \rightarrow[0,1]$ is a distortion function with $w(0)=0, w(1)=1$. Risk aversion is obtained if $w$ is convex (Yaari 1987, Theorem 2).

Let us define the Yaari non-expected utility problem as the optimization problem (1) in which the objective function is the Yaari functional $V\left(X_{T}\right)$ given in (11) and the admissible set $\mathcal{A}$ is the set of payoffs that satisfy the budget constraint. This problem was recently solved by He \& Zhou (2011) under some assumptions regarding the auxiliary function $M(z):=w^{\prime}(1-z) \bar{F}_{S_{T}^{\star}}^{-1}(1-z)$. We recall their solution.

Proposition 3.4. Assume that $M(z)$, given above, is continuous and uni-modal on $(0,1)$ : there exists $\tilde{z} \in(0,1)$ such that $M$ is strictly increasing on $(0, \tilde{z})$ and strictly decreasing on $(\tilde{z}, 1)$. The optimal payoff $X_{T}^{\star}$ solving

$$
\max _{c\left(X_{T}\right) \leqslant W_{0}, X_{T} \geqslant 0} \int_{0}^{+\infty} w\left[\mathbb{P}\left\{X_{T}>y\right\}\right] \mathrm{d} y
$$

is given as

$$
X_{T}^{\star}=\frac{W_{0}}{c\left(\mathbb{1}_{\left\{\underline{S}^{\star}<S_{T}^{\star}\right\}}\right)} \cdot \mathbb{1}_{\left\{\underline{S}^{\star}<S_{T}^{\star}\right\}},
$$

where $\underline{S}^{\star}>0$ is the zero of the function ${ }^{8}$

$$
Z(S)=\frac{1}{S} w\left(\bar{F}_{S_{T}^{\star}}(S)\right)+w^{\prime}\left(\bar{F}_{S_{T}^{\star}}(S)\right) \int_{S}^{+\infty} \frac{1}{t} \mathrm{~d} \bar{F}_{S_{T}^{\star}}(t), \quad S \leqslant \bar{F}_{S_{T}^{\star}}^{-1}(1-\tilde{z}) .
$$

The results of our numerical procedure are displayed in Figure 5. Here we use the market parameters of Table 1 and similarly to those in the example of He \& Zhou (2011), we take the distortion $w(x)=x^{\gamma}$ with $\gamma>1$ (proportional hazard model). In Panel 5(a) we take the proportional hazard parameter $\gamma=1$.1. We observe again a very good correspondence between the numerical solution and the exact one (it can be verified that the condition of Proposition 12 is satisfied). The initial guess yields a value for the Yaari objective function (distorted expectation) that is equal to 100.3765. Our algorithm constructs a payoff with objective value 101.4449, which is very close to the optimal value of 101.4454. In Panel 5(b) we increase the proportional hazard parameter to $\gamma=1.2$ (all other parameters

\footnotetext{
${ }^{8}$ Given a distribution function $F_{X}(x)$ we denote its survival function by $\bar{F}_{X}(x)=1-F_{X}(x)$.
} 
remain unchanged). The initial wealth distribution (dotted) has a distorted expectation of 99.9026. Our algorithm constructs a payoff with objective value equal to 100.4674 , whereas the optimal value 100.4663 .

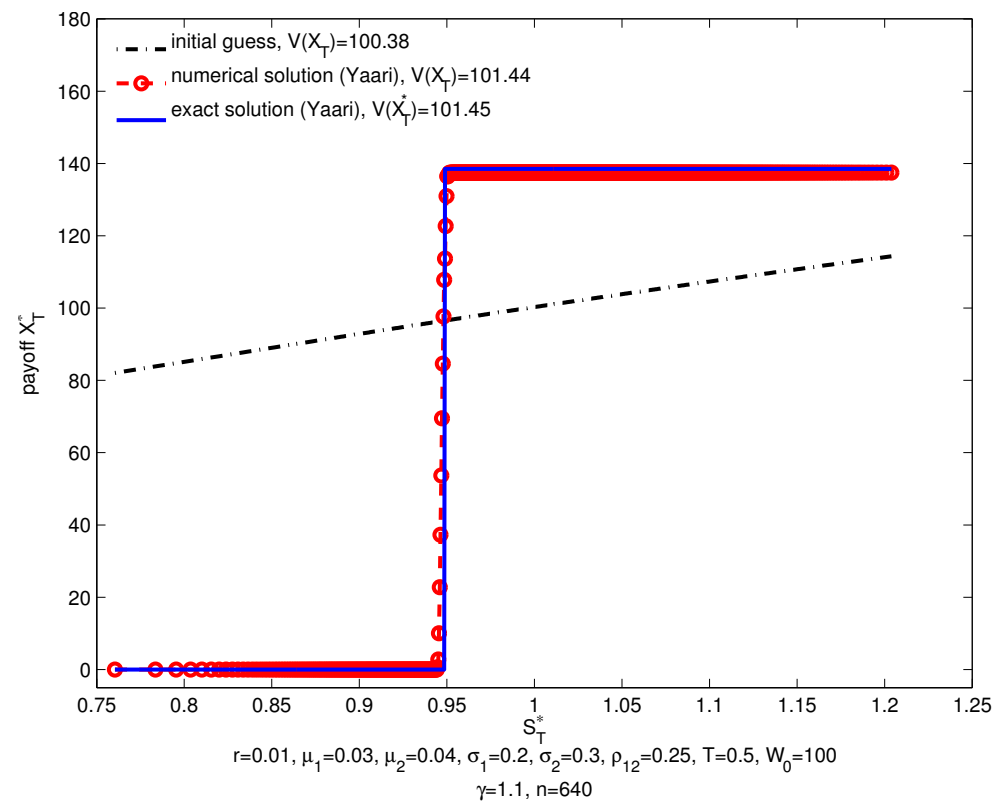

(a) $\gamma=1.1$

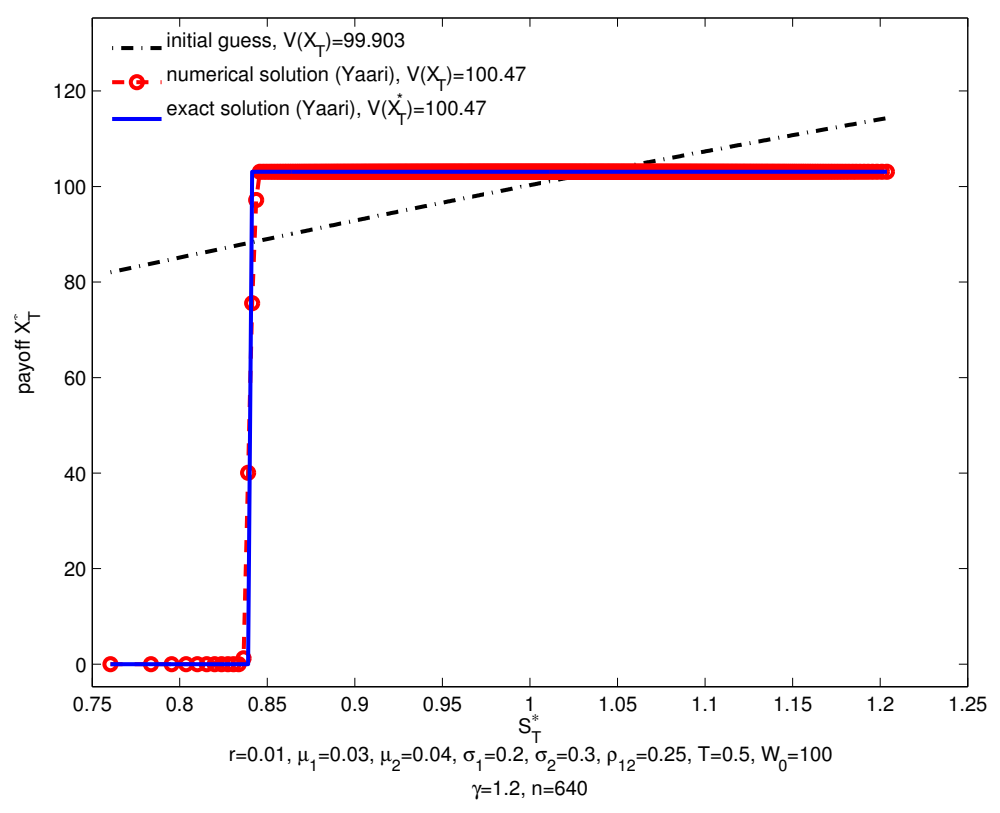

(b) $\gamma=1.2$

Figure 5: Optimal portfolios under Yaari's dual theory. 


\subsection{Additional evidence of the performance of the algorithm}

The examples in Section 3.2 illustrate, numerically and graphically, that Algorithm PA-SIP appears suitable for solving optimal portfolio problems under law-invariant preferences, i.e., in all examples the numerical and theoretical solutions match closely. In this section we further analyze the performance of the algorithm.

Convergence: We analyze the distance between the intermediate solutions and the analytical solutions and assess the extent to which they show subsequent improvements. In order to obtain a meaningful comparison, we first extend the subsequent approximations $X_{k, T}^{*}(k=0, \ldots, K)$ to a common base, which corresponds to the finest discretization of GOP; i.e., we consider $n_{K}$ equiprobable values in $\mathbf{s}_{K}^{\star}$ and interpolate and extrapolate the intermediate solutions $X_{k, T}^{*}(k=1,2, \ldots, K)$ using these $n_{K}$ discretization points. For ease of presentation we do not introduce a new notation for these extended versions of the solutions. The payoff-wise resp. objective-wise distance between a numerically obtained intermediate solution and the true analytical solution $X_{T}^{\star}$ is defined, for $k=0,1, \ldots, K$, as

$$
\delta_{k}^{K}(X):=\frac{\sqrt{\mathbb{E}\left[\left(X_{k, T}^{*}-X_{T}^{\star}\right)^{2}\right]}}{\sqrt{\mathbb{E}\left[\left(X_{T}^{\star}\right)^{2}\right]}} \quad \operatorname{resp} . \quad \delta_{k}^{K}(V):=\frac{\left|V\left(X_{k, T}^{*}\right)-V\left(X_{T}^{\star}\right)\right|}{\left|V\left(X_{T}^{\star}\right)\right|} .
$$

In Figure 6, we plot for all discussed applications the distances (on log-scale) as a function of the number $k=1,2, \ldots, K$.

We observe that in all cases the algorithm yields intermediate solutions that gradually improve. We stress that this feature is not an obvious point and is achieved thanks to the subsequent refinement of the initial point $X_{k, T}^{(0)}$, as described in Section 3.1 . If one naively uses the same starting point and runs the algorithm with a larger number of discretization points, then, according to our observations, the performance ultimately deteriorates because the number of unknown variables in the algorithm increases and the optimization problem fed into the solver fmincon is of larger dimensions. In our case, the distance decreases in all of the examples studied above and show close correspondence between theoretical and numerical solutions. In this regard, note that the objective-wise distance $\delta_{k}^{K}(V)$ appears to yield a better performance than the payoff-wise distance $\delta_{k}^{K}(X)$. However, the objective-wise distance $\delta_{k}^{K}(X)$ is the one explicitly being minimized in the procedure. As the objective-wise distance gets smaller, the solution gets closer to the true solution, which in turn decreases $\delta_{k}^{K}(X)$, but not necessarily with the same (relative) amount. Moreover, the objective-wise distance $\delta_{k}^{K}(V)$ involves the $L_{1}$ norm whereas the payoff-wise distance 
$\delta_{k}^{K}(X)$ used the $L_{2}$ norm? 9

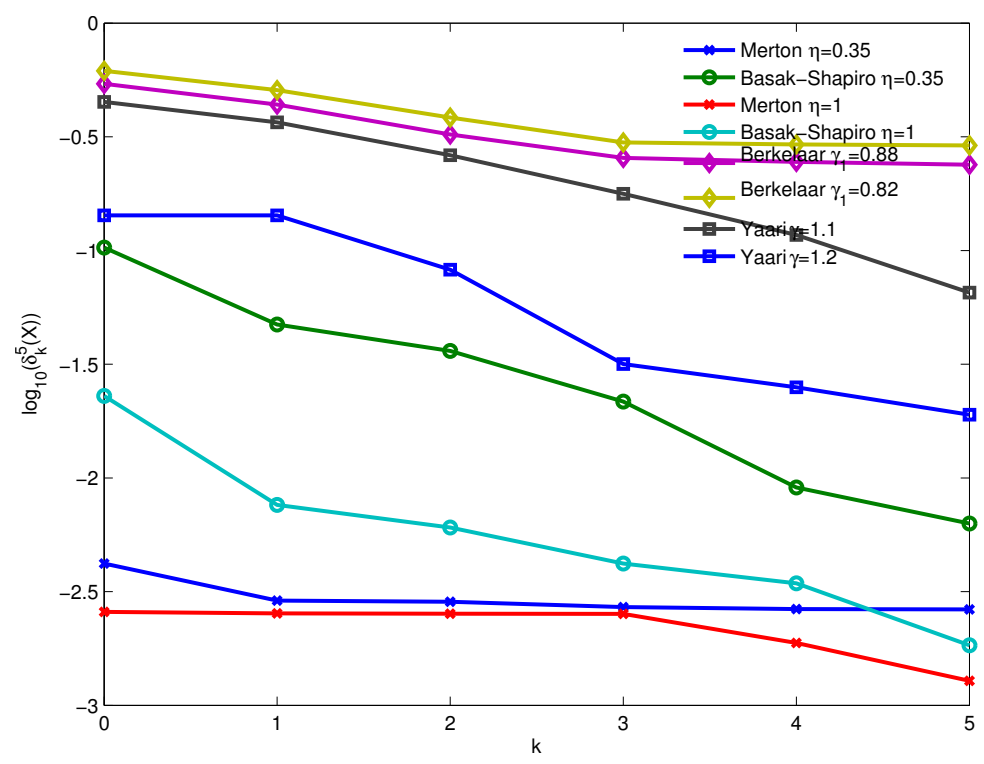

(a) payoff-metric

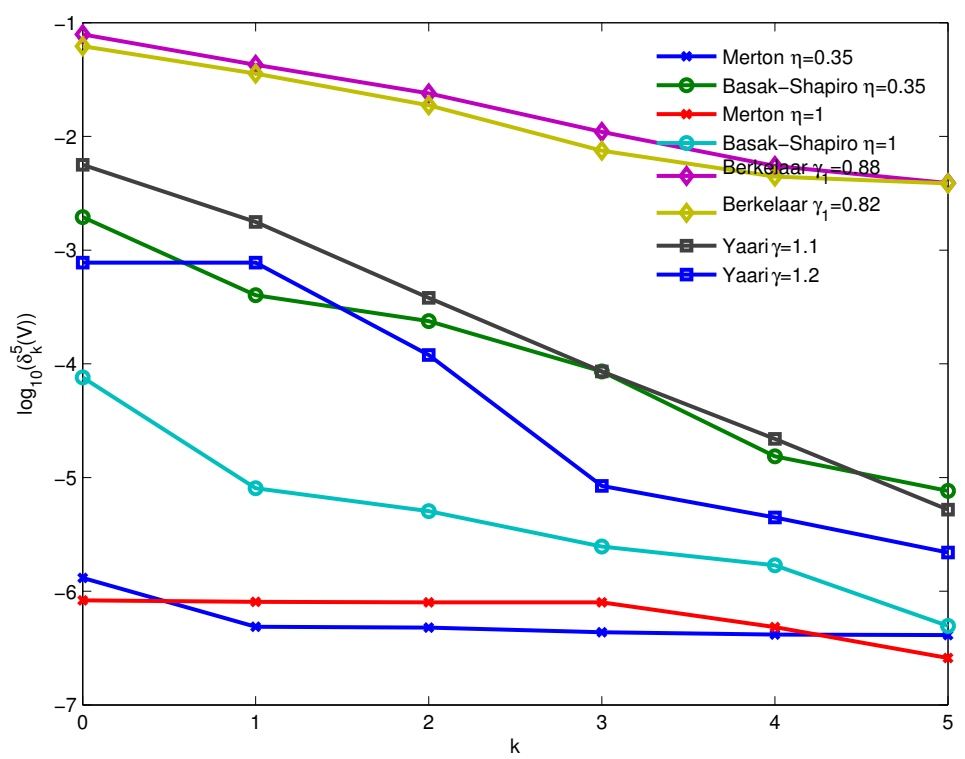

(b) objective-metric

Figure 6: Performance metrics of the algorithm.

Through extensive performance tests of our proposed algorithm, we also confirmed that the property that the solution must be monotonic is key for the algorithm to converge to the optimal portfolio. If the monotonicity property is not imposed upfront by reformu-

\footnotetext{
${ }^{9}$ In fact, if one would define the payoff-wise distance as $\widetilde{\delta}_{k}^{K}(X):=\frac{\mathbb{E}\left[\left|X_{k, T}^{*}-X_{T}^{\star}\right|\right]}{\mathbb{E}\left[\left|X_{T}^{\star}\right|\right]}$, one would observe that its magnitude in all eamples becomes much smaller.
} 
lating the problem with the unknown non-negative variables $\left(y_{1}, y_{2}, \ldots, y_{n}\right)$ instead of the unknown variables $\left(x_{1}, x_{2}, \ldots, x_{n}\right)$, the optimizer fmincon often misses the optimal solution and may even converge to a candidate solution that does not satisfy the monotonicity property or does not satisfy the constraints of the problem.

Sensitivity analysis: We conclude this section with a short sensitivity analysis to provide a comparison of the performance of the algorithm across various parameters. To do so, we perturb the base case of parameters (see Table 1), and check the impact on the performance of the algorithm. The parameters $\mu_{1}$ and $\sigma_{1}$ are first changed in such a way that the market price of risk, i.e., $\frac{\mu_{1}-r}{\sigma_{1}}=0.1$ remains the same (cases (2) and (3) in Tables 2 and 3 hereafter) and then to a higher value (cases (4) and (5)). We also assess the impact of dependence by using all parameters from the base case except the correlation level $\rho$ (Cases (6) and (7)).

Next, we assess for all these seven cases the impact on the solutions obtained for the Basak-Sahpiro VaR constrained problem (Section 3.2.2) and the Berkelaar optimization problem (Section ), respectively, using the payoff-wise distance $\delta_{k}^{K}(X)$ and objective-wise distance $\delta_{k}^{K}(V)(k=2,5)$ as the yardstick. The results are reported in Table 2 and Table 3. respectively. We find that the algorithm performs consistently well across various parameter ranges. The algorithm thus proves to be very useful, as it makes it possible to deal with any law-invariant problem, specifically those for which we do not know the analytical solution up front. Furthermore, this algorithm enables to potentially guess the analytical solution and to prove its optimality. Finally, the algorithm plays a key role in the following solutions to state-dependent optimal portfolio problems. As we will see in the next section, these problems are challenging, but very natural to consider, as individuals' preferences tend to be impacted by economic states (i.e., investors may not have the same risk aversion when the market is under stress as when the market is in normal conditions). Nevertheless, very few such optimization problems are solved explicitly in the literature; our algorithm provides a method for doing so. 
Table 2: Analysis of the Performance of Algorithm PA-SIP, case Basak-Shapiro (log preferences and $\underline{W}=92.5, \alpha=0.085$ )

\begin{tabular}{crrrr}
\hline & $\delta_{2}^{5}(X)$ & $\delta_{2}^{5}(V)$ & $\delta_{5}^{5}(X)$ & $\delta_{5}^{5}(V)$ \\
\hline \hline (1) Base case (Table $\mathbf{1}$ & $\mathbf{0 . 0 0 7 3}$ & $\mathbf{0 . 7 3 9 3 e - 4}$ & $\mathbf{0 . 0 0 2 3}$ & $\mathbf{0 . 0 1 3 1 e - 4}$ \\
\hline$(2) \lambda_{\bullet}=0.1, \sigma_{1}=0.3, \mu_{1}=0.04$ & 0.0073 & $0.0729 \mathrm{e}-4$ & 0.0023 & $0.0125 \mathrm{e}-4$ \\
\hline$(3) \lambda_{\bullet}=0.1, \sigma_{1}=0.1, \mu_{1}=0.02$ & 0.0073 & $0.0730 \mathrm{e}-4$ & 0.0023 & $0.0131 \mathrm{e}-4$ \\
\hline$(4) \lambda_{\bullet}=0.2, \sigma_{1}=0.3, \mu_{1}=0.07$ & 0.0261 & $0.1168 \mathrm{e}-3$ & 0.0066 & $0.0184 \mathrm{e}-3$ \\
\hline$(5) \lambda_{\bullet}=0.2, \sigma_{1}=0.1, \mu_{1}=0.03$ & 0.0261 & $0.1167 \mathrm{e}-3$ & 0.0067 & $0.0185 \mathrm{e}-3$ \\
\hline$(6) \rho=0.5$ & 0.0055 & $0.0416 \mathrm{e}-4$ & 0.0047 & $0.0320 \mathrm{e}-4$ \\
\hline$(7) \rho=0.1$ & 0.0086 & $0.1028 \mathrm{e}-4$ & 0.0025 & $0.0159 \mathrm{e}-4$ \\
\hline
\end{tabular}

Table 3: Analysis of the Performance of Algorithm PA-SIP, case Berkelaar $\left(\gamma_{1}=0.82\right)$

\begin{tabular}{ccccc}
\hline & $\delta_{2}^{5}(X)$ & $\delta_{2}^{5}(V)$ & $\delta_{5}^{5}(X)$ & $\delta_{5}^{5}(V)$ \\
\hline \hline (1) Base case (Table $\mathbf{1})$ & $\mathbf{0 . 4 0 1 2}$ & $\mathbf{0 . 0 3 1 2}$ & $\mathbf{0 . 2 0 5 1}$ & $\mathbf{0 . 0 1 4 8}$ \\
\hline (2) $\sigma_{1}=0.3, \mu_{1}=0.04$ & 0.4012 & 0.0312 & 0.1729 & 0.0116 \\
\hline (3) $\sigma_{1}=0.1, \mu_{1}=0.02$ & 0.4012 & 0.0312 & 0.2051 & 0.0148 \\
\hline (4) $\sigma_{1}=0.3, \mu_{1}=0.07$ & 0.7412 & 0.1246 & 0.4271 & 0.0248 \\
\hline (5) $\sigma_{1}=0.1, \mu_{1}=0.03$ & 0.7412 & 0.1246 & 0.3613 & 0.0172 \\
\hline$(6) \rho=0.5$ & 0.3583 & 0.0258 & 0.1746 & 0.0198 \\
\hline (7) $\rho=0.1$ & 0.4852 & 0.0425 & 0.2085 & 0.0108 \\
\hline
\end{tabular}




\section{Optimal Portfolios for State-Dependent Preferences}

Optimal portfolios for investors with law-invariant preferences have the property that the worst outcomes are obtained when the market declines. This feature does not correspond to the observation that investors may value a dollar that is received during economic recession more than when one received during economic expansion. The fact that investors care about the states in which they receive money is confirmed by the widespread practice according to which fund managers aim at tracking or beating a certain benchmark portfolio. Investment decisions can also be driven by market signals, such as changes in volatility and interest rates, or influenced by the performance of competitors.

To model state-dependent preferences, we use benchmarks. Specifically, we denote by $A$ the benchmark, i.e., a vector of random variables, that influences the investor's investment decision (e.g., stock or volatility indices, interest rates, asset prices or competitors' portfolios). The state-dependent investment problem can be formulated as follows:

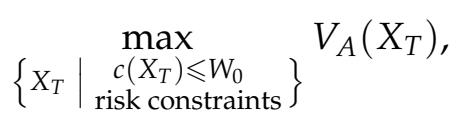

where the objective $V_{A}$ and the risk constraints may all depend on the random vector $A$.

Theorem 4.1 hereafter characterizes solutions to the state-dependent problem (14). In particular, it is shown that the solutions can be considered as increasing in the GOP, conditionally on the benchmark. This characterization significantly reduces the dimensionality of problem (14) and makes it possible to extend Algorithm PA-SIP to accommodate statedependent constraints and to solve complex state-dependent investment problems.

Theorem 4.1 (Optimal strategies). Let $A$ be a d-dimensional random vector and assume that $F_{S_{T}^{\star} \mid A}$ is continuously distributed. Assume that the objective function $V_{A}$ and risk constraints are non-decreasing ${ }^{10}$ and law-invariant, conditionally on $A 1^{11}$ If there exists a solution $X_{T}$ to problem (14), then there exists $X_{T}^{\star}$, which is increasing in $S_{T}^{*}$ conditionally on $A$ and also solves problem (14).

Proof. Let $X_{T}$ be a solution. It follows from Lemma (A.2) in Bernard et al. (2015b) that $U=F_{S_{T}^{\star} \mid A}\left(S_{T}^{\star}\right)$ is uniformly distributed on $(0,1)$, stochastically independent of $A$ and increasing in $S_{T}^{\star}$ conditionally on $A$. Consider the payoff $Y_{T}=F_{X_{T} \mid A}^{-1}(U)$. Invoking Lemma

\footnotetext{
${ }^{10}$ When $X \leqslant Y$, almost surely, then i) $V_{A}(X) \leqslant V_{A}(Y)$ and ii) if $X$ meets the risk constraints, then $Y$ also meets the risk constraints.

${ }^{11}$ If $F_{X \mid A}=F_{Y \mid A}$, then i) $V_{A}(X)=V_{A}(Y)$ and ii) if $X$ meets the risk constraints, then $Y$ also meets the risk constraints.
} 
(A.2) in Bernard et al. (2015b) again, $\left(X_{T}, A\right) \sim\left(Y_{T}, A\right)$ and

$$
\begin{aligned}
c\left(X_{T}\right)=\mathbb{E}\left[X_{T} \xi_{T}\right] & =\mathbb{E}\left[\mathbb{E}\left[X_{T} \xi_{T} \mid A\right]\right] \\
& \geqslant \mathbb{E}\left[\mathbb{E}\left[F_{X_{T} \mid A}^{-1}(U) \xi_{T} \mid A\right]\right] \\
& =\mathbb{E}\left[F_{X_{T} \mid A}^{-1}(U) \xi_{T}\right]=c\left(Y_{T}\right),
\end{aligned}
$$

where the inequality follows from the Frechet-Hoeffding bounds and from the fact that $F_{X_{T} \mid A}^{-1}(U)$ and $S_{T}^{\star}$ are comonotonic, conditionally on $A$. Since $\left(X_{T}, A\right) \sim\left(Y_{T}, A\right)$, it follows that $Y_{T}$ satisfies the risk constraints, and that $V_{A}\left(Y_{T}\right)=V_{A}\left(X_{T}\right)$ (conditional lawinvariance). Furthermore, there exists a non-negative constant $a$ such that $X_{T}^{\star}:=Y_{T}+a$ has cost $W_{0}$. The payoff $X_{T}^{\star}$ also satisfies the risk constraints, and $V_{A}\left(X_{T}^{\star}\right) \geqslant V_{A}\left(Y_{T}\right)$ (increasing preferences). Hence, $X_{T}^{\star}$ is at least as good as $X_{T}$; note also that it is increasing in $S_{T}^{*}$ conditionally on $A$ and also solves problem (14).

From the proof, every admissible solution $X_{T}$ to Problem (14) can be improved by a payoff $X_{T}^{\star}$ that is increasing in the GOP, i.e., $S_{T}^{\star}$, conditionally on $A$. Furthermore, denote by $G$ the joint distribution of $\left(X_{T}^{\star}, A\right)$. One has that $X_{T}^{\star}$ solves the problem

$$
\min _{X_{T} \mid\left(X_{T}, A\right) \sim G} c\left(X_{T}\right),
$$

i.e., $X_{T}^{\star}$ is the cheapest possible payoff having joint distribution $G$ with the benchmark $A$. The solution to this cost-efficiency problem was first provided in Theorem 3.4 of Bernard et al. (2015b); see also Theorem 4.4 in Bernard \& Tang (2016).

\subsection{Optimal Portfolio Choice Algorithm (State-Dependent Preferences)}

In this section, we outline the algorithm for solving the state-dependent problem (14). For ease of exposition, we ignore the potential presence of state-dependent risk constraints.

Set-up: Similarly as in the case with law invariant preferences (Section 3.1), we discretize the problem. The benchmark $A$ may have a discrete distribution with $\mathbb{P}\left[A=a_{j}\right]=$ $p_{j}$ for $j=1, \ldots, m$ (so $p_{j}>0$ and $\sum_{j=1}^{m} p_{j}=1$ ), and when $A$ is continuously distributed, we discretize it using an equiprobable sample of $m$ realizations, i.e., $a_{j}:=F_{A}^{-1}\left(\frac{j-0.5}{m}\right), j=$ $1,2, \ldots, m$ meaning that in this instance $p_{j} \equiv 1 / m$.

We consider $n m$ states with corresponding realizations of the random couple $\left(A, S_{T}^{\star}\right)$, which are obtained as follows. Consider the $m$ outcomes from the benchmark $A$, i.e., $a_{1}<$ $\ldots<a_{m}$. For each value $a_{j}$ of the benchmark, we simulate $n$ equiprobable values of the 
GOP $S_{T}^{\star}$, i.e., $s_{i j}^{\star}:=F_{S_{T}^{\star} \mid A=a_{j}}^{-1}\left(\frac{i-0.5}{n}\right), i=1,2, \ldots, n ; j=1,2, \ldots, m$. In this way, we obtain the following matrix:

$$
\left[\begin{array}{cccc}
\left(a_{1}, s_{11}^{\star}\right) & \left(a_{2}, s_{12}^{\star}\right) & \cdots & \left(a_{m}, s_{1 m}^{\star}\right) \\
\left(a_{1}, s_{21}^{\star}\right) & \left(a_{2}, s_{22}^{\star}\right) & \cdots & \left(a_{m}, s_{2 m}^{\star}\right) \\
\vdots & \vdots & \ddots & \vdots \\
\left(a_{1}, s_{n 1}^{\star}\right) & \left(a_{2}, s_{n 2}^{\star}\right) & \cdots & \left(a_{m}, s_{n m}^{\star}\right)
\end{array}\right] .
$$

All of the elements in a given column $j$ are equiprobable with probability $\frac{p_{j}}{n}$. Without loss of generality, we assume that for each $j=1, \ldots, m$, the $n$ realizations of the GOP are ordered as $s_{1 j}^{\star}<s_{2 j}^{\star}<\cdots<s_{n j}^{\star}$. Let $x_{i j}^{*}$ be the corresponding optimal wealth value in each of the $\mathrm{nm}$ states. The discrete formulation of the optimization problem reads as

$$
\max _{\left(x_{i j}\right)_{i, j} \in \mathcal{A}^{d}} V_{\mathbf{a}}\left(x_{i j}\right)
$$

with $\mathbf{a}:=\left(a_{1}, a_{2}, \ldots, a_{m}\right)$ and the admissible set $\mathcal{A}^{d}$ given as

$$
\mathcal{A}^{d}:=\left\{\left(x_{i j}\right) \in \mathbb{R}^{n m} \mid \frac{1}{n} \sum_{i=1}^{n} \sum_{j=1}^{m} \frac{x_{i j}}{s_{i j}^{\star}} p_{j} \leqslant W_{0}\right\} .
$$

In a similar way as in the case of state-independent problems, the optimization problem (15) suffers from the very large number of unknown variables (here, $n m$ unknown variables $x_{i j}$ ). However, from Theorem 4.1 the optimal portfolio must be conditionally increasing in the GOP (Theorem 4.1), and this makes it possible to reduce the dimensionality of the optimization problem (15a). Specifically, we formulate the following proposition.

Proposition 4.2. For each $j=1, \ldots, m$, the optimal wealth levels $x_{1 j}^{*}, x_{2 j}^{*}, \ldots, x_{n j}^{*}$ of the optimization problem 15 above are such that $x_{1 j}^{*} \leqslant x_{2 j}^{*} \leqslant \cdots \leqslant x_{n j}^{*}$.

To make use of the result in Proposition 4.2, we introduce auxiliary variables $y_{i j}=$ $x_{i j}-x_{(i-1) j}$ with the convention that $x_{0 j}=0$ and $i=1,2, \ldots, n$. The admissible set then writes as

$$
\mathcal{B}:=\left\{\left(y_{i j}\right) \in \mathbb{R}_{+}^{n m} \mid \sum_{i=1}^{n} \sum_{j=1}^{m} \zeta_{i j} y_{i j} \leqslant W_{0}\right\}, \quad \zeta_{i j}=\frac{p_{j}}{n} \sum_{k=i}^{n} \frac{1}{s_{k j}^{\star}} .
$$

By considering the admissible set (15b]) instead of [15b), we look for a solution in a space that is bounded from below. The optimization routine is more stable and converges better. Given the considerable number of variables, it is of utmost importance to construct the initial guess for the algorithm as closely as possible to the optimum in order to have a 
chance that the optimization procedure over $n m$ unknown variables will converge to the global optimal solution when $n$ increases 12

\section{Description of the algorithm:}

Step 1: We construct the best solution for an initial level of discretization $n_{0}$, say $n_{0}=$ 20. We split the optimization problem (15a) into solving $m$ problems for law-invariant preferences. Specifically, for each $j=1,2, \ldots, m$ we solve on a coarse grid of size $n_{0}=20$ and with (conditional) budget $W_{-1, j}^{\star}=W_{0}$ the optimization problem (15a), conditionally on $A=a_{j}$. To do so, we use $m$ times Algorithm PA-SIP (each involving an optimization over $n_{0}$ unknowns) and obtain the $m$ (conditional) optima $X_{0, j^{\prime}}^{\star} j=1,2, \ldots, m$. Note that the choice of taking all conditional budgets $W_{-1, j}$ equal to each other is arbitrary, but natural in that there is a priori no reason to justify differences among the conditional budgets according to the different values taken by the benchmark $A$. The $m$ (conditional) optima $X_{0, j}^{\star}$, $j=1,2, \ldots, m$ are next combined to obtain an initial (unconditional) guess regarding the optimal terminal wealth, which we feed into the global optimization of (15a) with $n_{0} m$ unknown variables. We rerun the optimization until the objective value no longer improves (with a given tolerance level at machine precision), and we thus obtain our best solution $X_{0, T}^{\star}$ for this level of discretization. From the resulting optimum $X_{0, T}^{\star}$, we can determine the conditional budgets $W_{0, j}^{\star}$ using the following decomposition formula:

$$
c\left(X_{T}^{\star}\right)=\mathbb{E}\left[\frac{X_{T}^{\star}}{S_{T}^{\star}}\right]=\mathbb{E}\left[\mathbb{E}\left[\frac{X_{T}^{\star}}{S_{T}^{\star}} \mid A\right]\right]=\sum_{j=1}^{m} \underbrace{\mathbb{E}\left[\frac{X_{T}^{\star}}{S_{T}^{\star}} \mid A=a_{j}\right]}_{W_{0, j}^{\star}} \mathbb{P}\left(A=a_{j}\right) .
$$

We find that conditional budgets are updated from $W_{-1, j}^{\star}=W_{0}$ to $W_{0, j}^{\star}=\frac{1}{n_{0}} \sum_{i=1}^{n_{0}} \frac{x_{i j}^{\star}}{s_{i j}^{\star}}, j=$ $1,2, \ldots, m$.

Step 2: We refine the procedure sequentially by doubling the number of discretization points. Hence, we take $n_{1}=2 n_{0}$ and solve, on a refined grid, $m$ law-invariant problems, conditionally on $A=a_{j}$ with updated conditional budgets $W_{0, j}^{\star}(j=1,2, \ldots, m)$ and using the optimum $X_{0, T}^{\star}$ that we obtained after the first step (appropriately extended to the finer grid) to yield the (conditional) starting points. The optima that next result from application of Algorithm PA-SIP (with $n_{1}$ unknown variables) are again combined into a starting point of the global optimization of [15a) with $n_{1} m$ unknown variables. The resulting optimum $X_{1, T}^{\star}$ will exhibit new conditional budgets $W_{1, j}^{\star}$. This doubling procedure is repeated $K$ times, resulting in a numerical optimum $X_{K, T}^{\star}$ on $n_{K}=2^{K} n_{0}$ discretization points over $m$ states of the benchmark $A$ with conditional budgets $W_{K, j}^{\star}$ among them. A more complete

\footnotetext{
${ }^{12} \mathrm{~A}$ natural extension is to also increase $m$.
} 
layout of our routine in pseudo-code is provided in Algorithm PA-SDP.

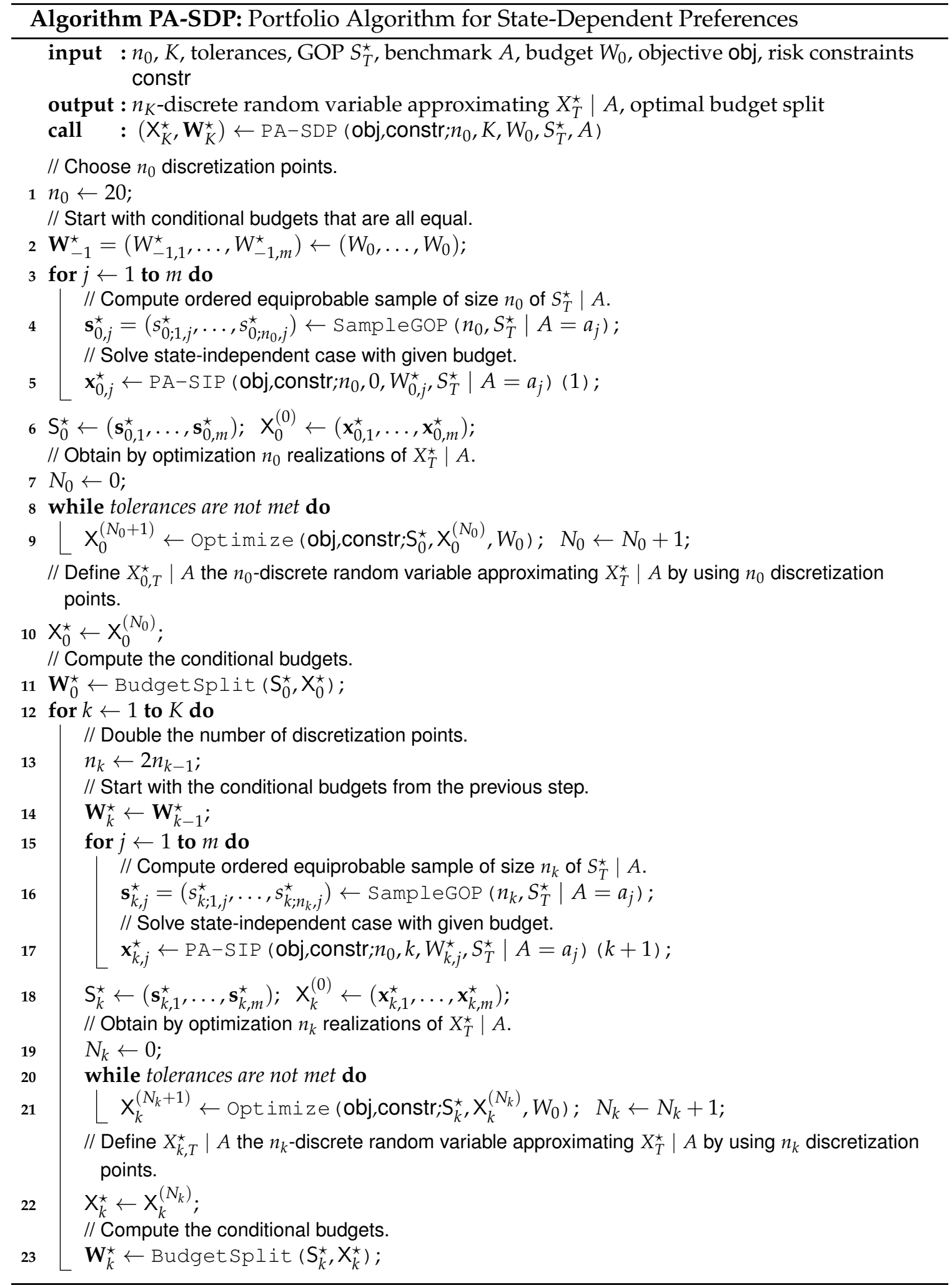




\subsection{Application of the Portfolio Algorithm for State-Dependent Preferences}

We provide a series of examples of portfolio choice problems with state-dependent features. Here, state-dependence may arise from a utility function that itself is state-dependent (Section 4.2.1) or from constraints imposed on the resulting optimal portfolio (Section 4.2.2).

\subsubsection{Merton type problems with state-dependent utility}

We examine two Merton-type problems with state-dependent utility functions that are of economic interest. In both cases analytical solutions are available and we are thus able to assess the performance of Algorithm PA-SDP in finding the solution. As for the benchmark, we consider the (discretized) GOP at some time $t<T$, i.e., we take $A=W_{0} S_{t}^{\star}$. Note that by varying the parameter $t$, we have the flexibility to let the benchmark $A$ take various forms. Moreover, joint sampling from $A$ and $S_{T}^{\star}$ is straightforward.

Example A. We consider the following expected utility maximization problem with a state-dependent utility function:

$$
\max _{c\left(X_{T}\right)=W_{0}} \mathbb{E}\left[u\left(A, X_{T}\right)\right], \quad u(a, x)=2 \sqrt{a x}
$$

The value of a payoff is thus essentially measured as the expectation of its (geometric) average with the benchmark $A$. The benchmark might be another portfolio of the investor, which she cannot adjust (e.g., a pension fund managed by her employer), but which clearly affects her future welfare. By taking the geometric average we consider the presence of this benchmark portfolio in a meaningful way.

Using pathwise optimization, it can be readily shown that the optimal solution to the stated optimization problem is given as $X_{T}^{\star}=\frac{1}{\theta^{2}} A S_{T}^{\star 2}$, where $\theta$ follows from the budget constraint (see Proposition 1 of Bernard et al. (2018)). Furthermore, since the conditions of Theorem 4.1 are fulfilled the use of Algorithm PA-SDP is justified, and a numerical solution can be obtained. In Figure 7, we present the numerical solution (for the level of discretization $m=2$ ) provided by the algorithm and contrast it with the exact solution. We observe that there is a close correspondence, which demonstrates the potential of the algorithm in solving state-dependent optimal portfolio selection problems.

Example B. We consider an expected utility maximization problem in which the statedependence arises from making the (Arrow-Pratt) risk aversion coefficient a function of a benchmark. It appears indeed intuitive that investors are more risk averse in bear markets than in bull markets; see e.g., Dong \& Sircar (2014), who let the risk aversion coefficient 


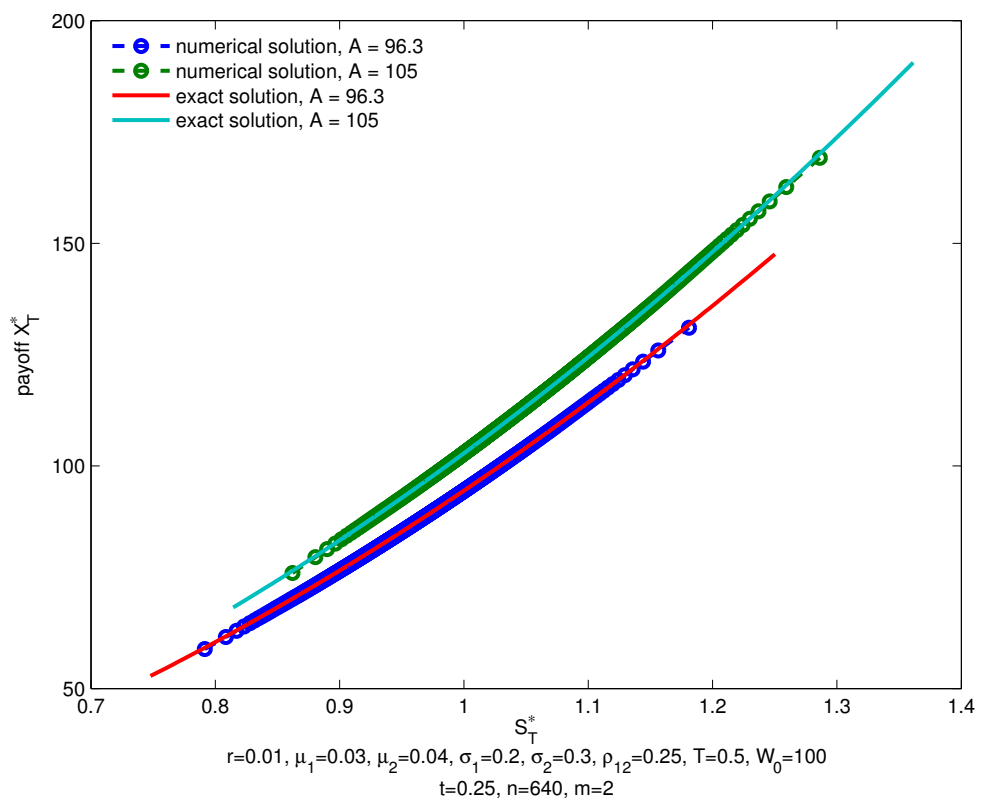

Figure 7: Merton example with $u(a, x)=2 \sqrt{a x}$.

depend on the value of the portfolio itself. As for the choice of utility function, we use the CRRA utility, and the optimization problem then reads as

$$
\max _{c\left(X_{T}\right)=W_{0}} \mathbb{E}\left[u\left(A, X_{T}\right)\right], \quad u(a, x)=\frac{x^{1-\eta(a)}}{1-\eta(a)}, \quad \eta(a)=2+22 \exp (-0.025 a) .
$$

Observe that the risk aversion coefficient $\eta(a)$ is decreasing in $a$, i.e., when the benchmark (which can be seen as an indicator of the strength of the economy) is high, the risk aversion coefficient is small. It decreases from 24 (when the benchmark value is close to 0 ) to 2 (when it goes to infinity). Using pathwise optimization we find that the theoretical solution to the stated optimization problem is given as $X_{T}^{\star}=\left(\theta S_{T}^{\star}\right)^{\frac{1}{\eta(A)}}$, in which $\theta$ follows from the budget constraint. Figure 8 displays, for the case $m=3$, the analytical and numerical solutions and contrasts them with state-independent solutions (SIP) that are obtained using a constant level of risk aversion $\eta(a)$.

We observe that when the benchmark $A$ is high, the optimal investment $X_{T}^{\star}$ yields high income, and that when $A$ is low, less income is received. We make two additional observations. First, we note that for small values of $A$, the optimal payoff is flatter than for higher values of $A$. In other words, the higher the risk aversion, the flatter the payoff of the optimal portfolio. This is intuitive, as with infinite risk aversion the optimal portfolio of a CRRA investor consists in allocating the investor's entire budget to the risk-free asset. Second, we observe that as compared to the state-independent case, the use of the state- 


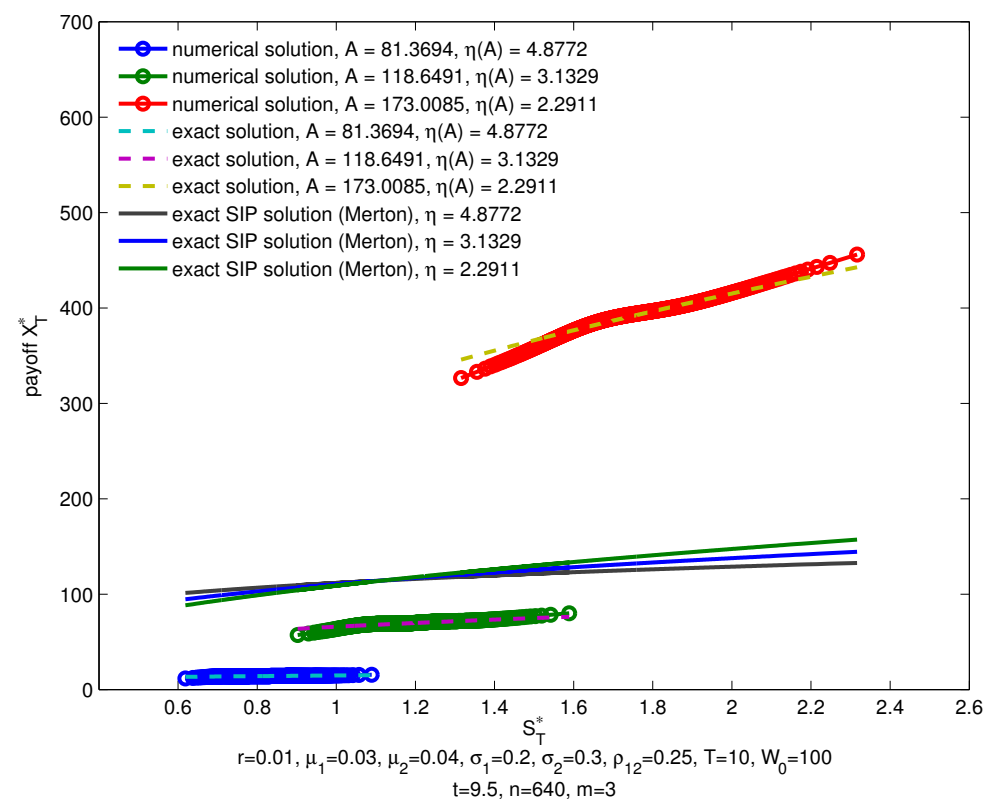

Figure 8: Merton example with power utility $u(a, x)=\frac{x^{1-\eta(a)}}{1-\eta(a)}$ and state-dependent risk aversion $\eta(a)=2+22 \exp (-0.025 a)$.

dependent utility function yields an optimal investment in which terminal wealth is very low when the benchmark is at the lowest. This feature is at odds with the belief that a more risk averse individual would be willing to purchase more protection and therefore to use a larger part of his budget for obtaining protection against the worst states of the market. In fact, this is the reason why we model $\eta(a)$ as a decreasing function in $a$; yet although this link between risk aversion coefficient and the state of the market appears natural, the resulting optimal behaviour of the investor is counter-intuitive and surprising.

Table 4: Standard deviation of the optimal portfolio when the utility function to optimize is the CRRA utility with a fixed risk aversion coefficient equal to $\eta$ (SIP) or with a statedependent risk aversion coefficient (SDP).

\begin{tabular}{cccc}
\hline $\operatorname{SIP} \eta=2.2911$ & $\operatorname{SIP} \eta=3.1329$ & $\operatorname{SIP} \eta=4.8772$ & $\operatorname{SDP} \eta(\cdot)$ \\
\hline 20.1421 & 14.4206 & 9.0833 & 166.5896 \\
\hline
\end{tabular}

The counter-intuitive behavior is also confirmed by analyzing the variances of the payoffs. In Table 4, we show that when the state-dependent utility function is used, the variance of the optimal payoff is larger than that of the payoff obtained in the stateindependent case ${ }^{13}$ One should use a fixed risk aversion coefficient of $\eta=0.4445$ in the

\footnotetext{
${ }^{13}$ In the case of state-independent preferences, there is a constant risk aversion $\eta$, and the results in Table 4
} 
state-independent case, to obtain the same standard deviation (namely 166.5896) as when using the state-dependent risk aversion.

Note that the procedure described in this section can handle very general state-dependent objectives. For instance, it can deal with the optimization of a loss averse utility function in which the reference level is linked to a stochastic benchmark $A$ :

$$
\max _{c\left(X_{T}\right)=W_{0}} \mathbb{E}\left[u\left(A, X_{T}\right)\right], \quad u(a, x)=\left\{\begin{array}{ll}
-C_{1}(a-x)^{\eta_{1}} & x \leqslant a \\
C_{2}(x-a)^{\eta_{2}} & x>a
\end{array} .\right.
$$

Comparing with Section 3.3 , we thus obtain an optimal choice problem for a loss-averse investor with a state-dependent reference level $A$. The investor thus considers positive deviations of his terminal wealth vis-à-vis the benchmark as gains and negative deviations as losses.

\subsubsection{Merton example with a CoVaR constraint}

In this final section, we propose to solve an optimal portfolio problem with a state-dependent risk constraint in order to illustrate our algorithm and to demonstrate how it can deal easily with additional state-dependent constraints. To do so, we consider the optimal investment for a company subject to a constraint on its CoVaR (systemic risk measure proposed by Adrian \& Brunnermeier (2011)). This example extends the optimization of expected utility with a VaR constraint (i.e., the probability constraint of Basak \& Shapiro (2001)) that we considered in Section 3.2.2. Now the VaR constraint is replaced by a CoVaR constraint, i.e., the VaR conditional on some events, such as a financial crisis (that we could model, for instance, as the GOP being lower than its VaR at 95\%, as in Bernard et al. (2015b)).

We thus consider a state-independent objective $V\left(X_{T}\right)$ where $X_{T}$ is the wealth at maturity $T$ (typically $V(X)=\mathbb{E}[u(X)]$ ), with an additional state-dependent risk constraint (CoVaR, for instance). The problem can be formulated as

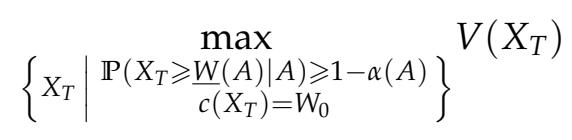

where $A$ is the benchmark.

are obtained explicitly. Indeed, recall that for the CRRA utility, the optimal wealth obtained with an initial budget $W_{0}$ is given by

$$
X_{T}^{\star}=W_{0} e^{\left(1-\frac{1}{\eta}\right) M_{T}} e^{-\frac{1}{2}\left(1-\frac{1}{\eta}\right)^{2} V_{T}}\left(S_{T}^{\star}\right)^{\frac{1}{\eta}},
$$

from which it follows that $\operatorname{Var}\left(X_{T}^{\star}\right)=W_{0}^{2} e^{2 M_{T}-\left(1-\frac{2}{\eta}\right) V_{T}}\left(e^{\frac{1}{\eta^{2}} V_{T}}-1\right)$. In the case of state-dependent preferences - that is, a varying risk aversion $\eta(\cdot)$ - the results in Table 4 are obtained numerically. 
As a specific example of a CoVaR constraint as it has been discussed in the literature, take $s_{q}:=F_{S_{T}^{\star}}^{-1}(q)$ the $q$-quantile of $S_{T}^{\star}(0<q<1)$ and let the event $\Gamma_{q}=\left\{S_{T}^{\star}<s_{q}\right\}$ represent a stressed situation. As benchmark we take $A=\mathbb{1}_{\Gamma_{q}}$, i.e., $A$ is the indicator of being in stress, that is, it takes $m=2$ values, namely $a_{1}=0$ (no stress) and $a_{2}=1$ (stress). The corresponding probabilities are then $p_{1}=\mathbb{P}\left[A=a_{1}\right]=1-q$ and $p_{2}=$ $\mathbb{P}\left[A=a_{2}\right]=\mathbb{P}\left[S_{T}^{\star}<s_{q}\right]=q$. Let us denote $\underline{W}_{j}=\underline{W}\left(a_{j}\right)$ and $\alpha_{j}=\alpha\left(a_{j}\right)$. For floor values of the benchmark we consider $\left(\underline{W}_{1}, \underline{W}_{2}\right)=(0, \underline{W})$, and for the probabilities we consider $\left(\alpha_{1}, \alpha_{2}\right)=(1, \alpha)$ so that there is effectively only one constraint (which will be active in a stress situation), i.e., $\mathbb{P}\left(X_{T} \geqslant \underline{W}_{2} \mid A=a_{2}\right)=\mathbb{P}\left(X_{T} \geqslant \underline{W} \mid \Gamma_{q}\right) \geqslant 1-\alpha_{1}=1-\alpha$. As for the objective in (17), we take $V(X)=\mathbb{E}\left[u_{\eta}(X)\right]$ with CRRA utility function $u_{\eta}$, as given in (5). We obtain the following constrained optimal portfolio problem

$$
\max _{\left\{X_{T} \mid \begin{array}{l}
\mathbb{P}\left(X_{T} \geqslant W \mid \Gamma_{q}\right) \geqslant 1-\alpha \\
c\left(X_{T}\right)=W_{0}
\end{array}\right.} \mathbb{E}\left[u_{\eta}\left(X_{T}\right)\right] .
$$

We apply Algorithm PA-SDP to solve this optimization problem numerically. First, we consider a risk aversion coefficient equal to $\eta=0.5$, a floor value $\underline{W}=95$ and a probability level $1-\alpha=0.9$ corresponding to the stress situation defined by $\Gamma_{0.125}=\left\{S_{T}^{\star}<0.91007\right\}$. The stress level is taken as its $12.5 \%$ quantile (which is typically much higher than the level recommended by regulators). This level facilitates the visualization of the constraint, as can be seen from the results shown in Figure 9(a). Next, we change the risk aversion level to $\eta=2$ and present the results in Figure 9(b).

We observe two parts in the optimal portfolio decisions, depending on whether we are in a stressed scenario (corresponding to the GOP being above or below $s=0.91007$, which is its $12.5 \%$ quantile). The state-independent (Merton) solution violates the CoVaR constraint. This can be seen from Figure 9, but is also numerically computed in Table 5 , Recall that the CoVaR constraint is only active during stress, in which case the payoff should be above $\underline{W}=95$, with probability equal to 0.9 . However, for a risk aversion coefficient $\eta=0.5$, resp. $\eta=2$ the Merton solution (SIP) has a probability equal to 0 resp. 0.2562 to remain above 95 in the stressed scenario. Our routine constructs a solution (SDP) that has a probability 0.9016 , resp. 0.9109 , to be above 95 , which is compliant with the required level $1-\alpha=0.9$.

To remedy the CoVaR violation of the Merton solution, we observe that in the stressed situation more insurance (consumption) will be purchased so that the desired protection is obtained. The more risk averse, the less extra insurance is needed to shift to the stressed situation. This result, in turn, is then compensated by a larger deviation of the payoff with 


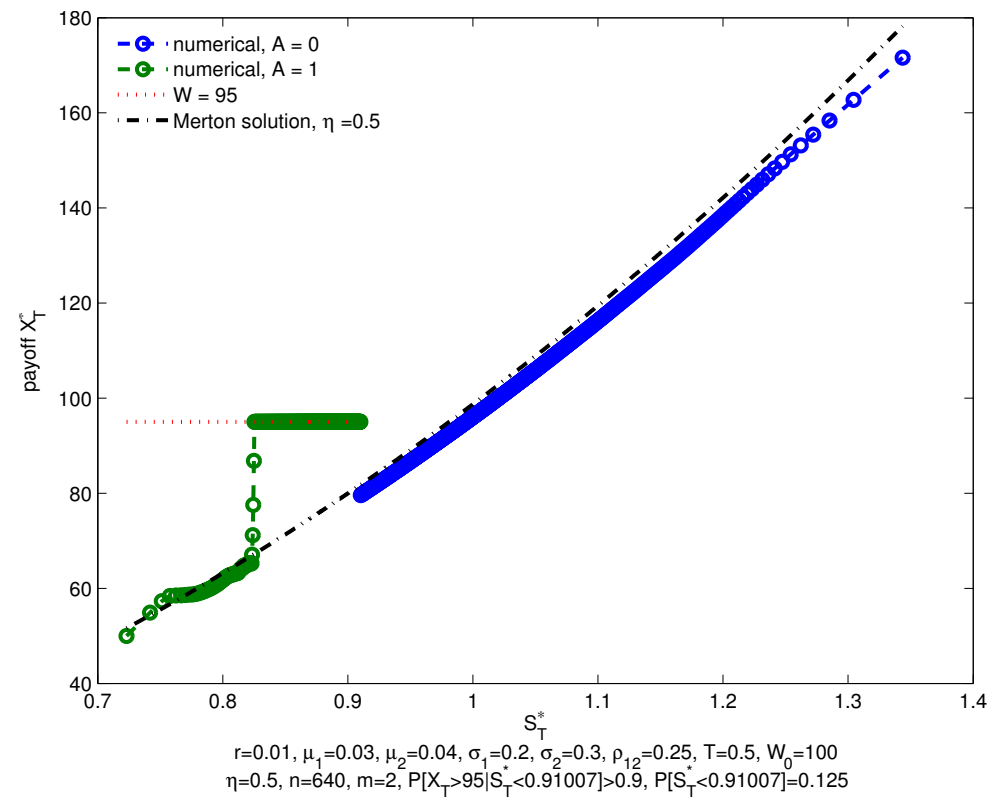

(a) $\eta=0.5$

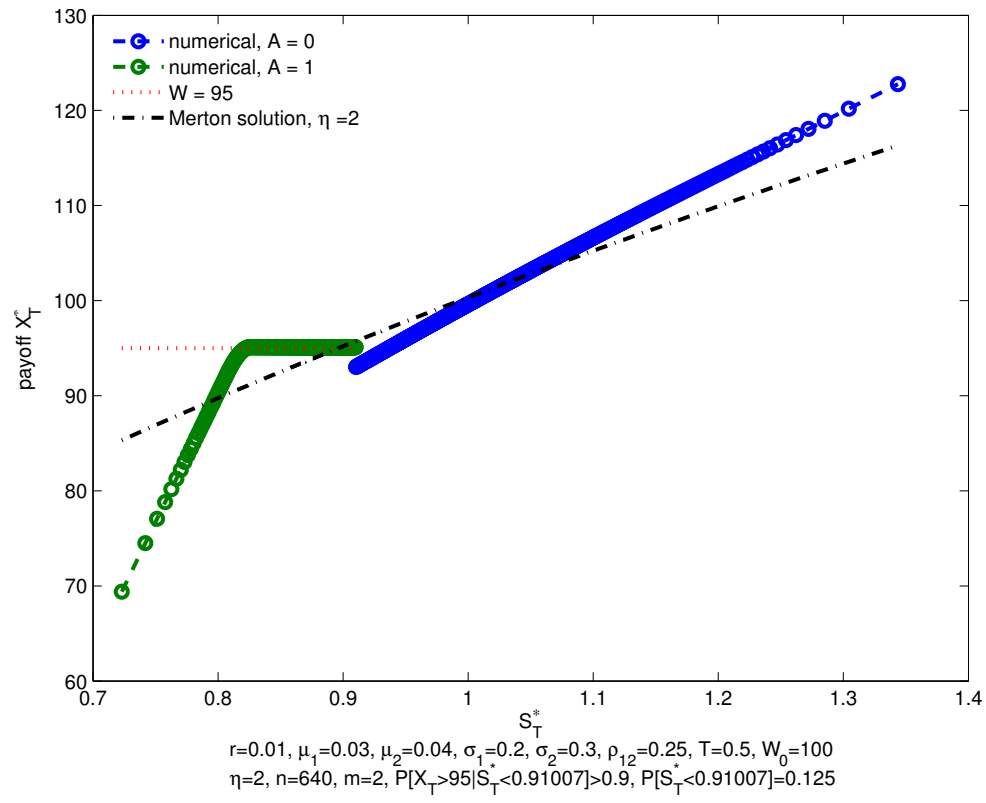

(b) $\eta=2$

Figure 9: CRRA utility with CoVaR constraint.

respect to the Merton solution; see Figure 9 . 
Table 5: CoVaR value $\mathbb{P}\left(X_{T} \geqslant \underline{W}(A) \mid A\right)$.

\begin{tabular}{ccccc}
\hline & $\mathrm{SDP} \eta=0.5$ & $\mathrm{SIP} \eta=0.5$ & $\mathrm{SDP} \eta=2$ & $\mathrm{SIP} \eta=2$ \\
\hline no stress $(A=0)$ & 0.6453 & 0.7125 & 0.9094 & 1 \\
stress $(A=1)$ & 0.9016 & 0 & 0.9109 & 0.2562 \\
\hline
\end{tabular}

\section{Conclusions}

Since the 1980s, it has been known that the payoff that generates a given distribution of terminal wealth at lowest possible cost must be increasing in the market asset (see Dybvig (1988), Bernard et al. (2014a) and Carlier \& Dana (2011)). However, this characterization of optimal payoffs has received little attention in the analysis and construction of solutions to optimal portfolio problems. In this paper, we exploit this monotonicity property to construct an efficient numerical method that makes it possible to first obtain optimal portfolios for a wide range of problems with law-invariant objectives, and then to extend these problems to include the case of state-dependent preferences and of additional risk constraints.

We apply our method to various classic (non-)expected utility problems for which explicit solutions are available, and we show that the numerical solutions are compatible with them. This observation allows us to conclude that we can use the algorithm to deal with portfolio optimization problems for which explicit solutions are not available. $\mathrm{Nu}$ merical examples were implemented in a Black-Scholes setting but more general market models can also be considered, such as Lévy markets with agents using Esscher pricing to value payoffs (Von Hammerstein et al. (2014), Rüschendorf \& Wolf (2015)).

We foresee a large number of additional applications of the algorithm developed in this paper, and we sketch a few of these. For instance, Tepla (2001) and Basak et al. (2006) explicitly derive optimal investment policies under minimum performance constraints in a Black-Scholes market setup. The algorithm that we propose makes it possible to extend these results to more general markets and to additional risk constraints. We may also extend the work of Cvitanić et al. (2003) to non-expected utility settings. There are, moreover, additional applications to insurance and pension fund allocation (see e.g., Cairns et al. (2006) or Deelstra et al. (2003)). Finally, in the literature, equilibrium problems have been studied in a law invariant setting (Lioui \& Poncet (2001), Basak \& Shapiro (2001)), but there are almost no studies dealing with equilibrium when agents have state-dependent preferences. We believe that our results are useful in studying such problems. 


\section{Acknowledgment}

C. Bernard gratefully acknowledges support from the project on Systemic Risk funded by the GRI in financial services, the Louis Bachelier Institute, and from the Odysseus research grant funded by Flanders Research Foundation. R. H. De Staelen acknowledges the support of the Flanders Research Foundation (FWO15/PDO/076). S. Vanduffel acknowledges the financial support of the Stewardship of Finance Chair and of the Flanders Research Foundation.

\section{References}

Adrian, T., \& Brunnermeier, M. K. (2011). CoVaR. Tech. rep., National Bureau of Economic Research.

Allais, M. (1953). Le Comportement de l'Homme Rationnel Devant le Risque: Critique des Axiomes et Postulats de l'école Américaine. Econometrica, 21(4), 503-546.

Basak, S., \& Shapiro, A. (2001). Value-at-risk-based risk management: optimal policies and asset prices. Review of Financial studies, 14(2), 371-405.

Basak, S., Shapiro, A., \& Teplá, L. (2006). Risk management with benchmarking. Management Science, 52(4), 542-557.

Berkelaar, A., Kouwenberg, R., \& Post, T. (2004). Optimal Portfolio Choice Under Loss Aversion. The Review of Economics and Statistics, 86(4), 973-987.

Bernard, C., Boyle, P. P., \& Vanduffel, S. (2014a). Explicit representation of cost-efficient strategies. Finance, 35(2), 5-55.

Bernard, C., Chen, J. S., \& Vanduffel, S. (2014b). Optimal portfolios under worst-case scenarios. Quantitative Finance, 14(4), 657-671.

Bernard, C., Chen, J. S., \& Vanduffel, S. (2015a). Rationalizing investors' choices. Journal of Mathematical Economics, 59, 10-23.

Bernard, C., Maj, M., \& Vanduffel, S. (2011). Improving the design of financial products in a multidimensional Black-Scholes market. North American Actuarial Journal, 15(1), 77-96.

Bernard, C., Moraux, F., Rüschendorf, L., \& Vanduffel, S. (2015b). Optimal payoffs under statedependent preferences. Quantitative Finance, 15(7), 1157-1173.

Bernard, C., \& Tang, J. (2016). Simplified hedge for path-dependent derivatives. International Journal of Theoretical and Applied Finance, 19(07), 1650045.

Bernard, C., \& Vanduffel, S. (2014). Financial bounds for insurance claims. Journal of Risk and Insurance, 81(1), 27-56.

Bernard, C., Vanduffel, S., \& Ye, J. (2018). Optimal portfolio under state-dependent expected utility. SSRN working paper.

Birnbaum, M. H. (1997). Violations of monotonicity in judgment and decision making. In A. A. J. Marley (Ed.) Choice, decision, and measurement: Essays in honor of R. Duncan Luce, (pp. 73-100). Mahwah, NJ:Erlbaum.

Birnbaum, M. H., \& Navarrette, J. (1998). Testing descriptive utility theories: Violations of stochastic dominance and cumulative independence. Journal of Risk and Uncertainty, 17, 49-78.

Björk, T. (2004). Arbitrage Theory in Continuous Time. Oxford University Press.

Browne, S. (1999). Reaching goals by a deadline: Digital options and continuous-time active portfolio management. Advances in Applied Probability, 31(2), 551-577.

Cairns, A., Blake, D., \& Dowd, K. (2006). Stochastic lifestyling: Optimal dynamic asset allocation for defined contribution pension plans. Journal of Economic Dynamics and Control, 30(5), 843-877.

Carlier, G., \& Dana, R.-A. (2011). Optimal demand for contingent claims when agents have lawinvariant utilities. Mathematical Finance, 21(2), 169-201. 
Cvitanić, J., Goukasian, L., \& Zapatero, F. (2003). Monte carlo computation of optimal portfolios in complete markets. Journal of Economic Dynamics and Control, 27(6), 971-986.

Daniel, K., Grinblatt, M., Titman, S., \& Wermers, R. (1997). Measuring mutual fund performance with characteristic-based benchmarks. Journal of finance, 52(3), 1035-1058.

d'Aspremont, A. (2004). Shape constrained optimization with applications in finance and engineering. Ph.D. thesis, Stanford University.

Deelstra, G., Grasselli, M., \& Koehl, P.-F. (2003). Optimal investment strategies in the presence of a minimum guarantee. Insurance: Mathematics and Economics, 33(1), 189-207.

Dentcheva, D. (2006). Probabilistic and Randomized Methods for Design under Uncertainty, chap. Optimization Models with Probabilistic Constraints, (pp. 49-97). London: Springer London.

Dong, Y., \& Sircar, R. (2014). Time-inconsistent portfolio investment problems. In Stochastic Analysis and Applications 2014, (pp. 239-281). Springer.

Dybvig, P. (1988). Inefficient dynamic portfolio strategies or how to throw away a million dollars in the stock market. Review of Financial Studies, 1(1), 67-88.

Edwards, W. (1955). The Prediction of Decisions Among Bets. Journal of Experimental Psychology, 50(3), 201-214.

Edwards, W. (1962). Subjective Probabilities Inferred from Decisions. Psych. Review, 69(2), 109-135.

Eeckhoudt, L., Gollier, C., \& Schlesinger, H. (2011). Economic and Financial Decisions under Risk. PUPress.

Floudas, C. A., \& Pardalos, P. M. (2009). Encyclopedia of Optimization. Springer US, 2nd ed.

Hanson, M. A. (1981). On sufficiency of the Kuhn-Tucker conditions. Journal of Mathematical Analysis and Applications, 80(2), 545-550.

Hanson, M. A. (1999). Invexity and the Kuhn-Tucker theorem. Journal of Mathematical Analysis and Applications, 236(2), 594-604.

He, X. D., \& Zhou, X. Y. (2011). Portfolio choice via quantiles. Mathematical Finance, 21(2), 203-231.

Inada, K.-I. (1963). On a two-sector model of economic growth: Comments and a generalization. Review of Economic Studies, 30(2), 119-127.

Kahneman, D., \& Tversky, A. (1979). Prospect theory: An analysis of decision under risk. Econometrica, 47(2), 263-291.

Levy, H. (2008). First degree stochastic dominance violations: decision weights and bounded rationality. Economic Journal, 118, 759-774.

Levy, H., \& Markowitz, H. M. (1979). Approximating expected utility by a function of mean and variance. The American Economic Review, 69(3), 308-317.

Lioui, A., \& Poncet, P. (2001). On optimal portfolio choice under stochastic interest rates. Journal of Economic Dynamics and Control, 25(11), 1841-1865.

Lopes, L. L. (1987). Between hope and fear: The psychology of risk. Advances in Experimental Social Psychology, 20, 255-295.

Lopes, L. L., \& Oden, G. C. (1999). The role of aspiration level in risky choice: A comparison of cumulative prospect theory and SP/A theory. Journal of Mathematical Psychology, 43(2), 286313.

Machina, M. J. (1987). Choice under uncertainty: Problems solved and unsolved. Economic Perspectives, 1(1), 121-154.

Machina, M. J. (1995). Non-expected utility and the robustness of the classical insurance paradigm. The Geneva Papers on Risk and Insurance Theory, 20, 9-50.

Machina, M. J. (2004). Nonexpected utility theory. In J. T. Teugels, \& B. Sundt (Eds.) Encyclopedia Of Actuarial Science, vol. 2, (pp. 1173-1179). John Wiley \& Sons, Ltd, Chichester.

Markowitz, H. M. (1952). Portfolio Selection. Journal of Finance, 7(1), 77-91.

Markowitz, H. M., Todd, G. P., \& Sharpe, W. F. (2000). Mean-Variance Analysis in Portfolio Choice and Capital Markets. Frank J. Fabozzi Series. Wiley.

MATLAB ${ }^{T M}$ (2013). The Mathworks, Inc., Natick, Massachusetts, R2013b (8.2.0.701) ed.

Merton, R. (1969). Lifetime Portfolio Selection under Uncertainty: The Continuous-Time Case. Review of Economics and Statistics, 51(3), 247-257.

Merton, R. (1971). Optimum consumption and portfolio rules in a continuous-time model. Journal of Economic Theory, 3, 373-413. 
Nocedal, J., \& Wright, S. (2006). Numerical Optimization. Springer Series in Operations Research and Financial Engineering. Springer-Verlag New York, 2nd edition ed.

Platen, E., \& Heath, D. (2006). A benchmark approach to quantitative finance. No. 13 in Springer Finance. Springer.

Quiggin, J. (1993). Generalized Expected Utility Theory - The Rank-Dependent Model. Kluwer.

Roll, R. (1992). A mean/variance analysis of tracking error. Journal of Portfolio Management, 18(4), $13-22$.

Rüschendorf, L., \& Wolf, V. (2015). Cost-efficiency in multivariate Lévy models. Dependence Modeling, 3(1).

Shefrin, H. M., \& Statman, M. (2000). Behavioral portfolio theory. Journal of Financial and Quantitative Analysis, 35(2), 127-151.

Tepla, L. (2001). Optimal investment with minimum performance constraints. Journal of Economic Dynamics and Control, 25(10), 1629-1645.

Tversky, A., \& Kahneman, D. (1992). Advances in prospect theory: Cumulative representation of uncertainty. Journal of Risk and Uncertainty, 5(4), 297-323.

Von Hammerstein, E. A., Lütkebohmert, E., Rüschendorf, L., \& Wolf, V. (2014). Optimality of payoffs in Lévy models. International Journal of Theoretical and Applied Finance, 17(6), 1450041.

von Neumann, J., \& Morgenstern, O. (1947). Theory of Games and Economic Behavior. PUPressx.

Yaari, M. (1987). The Dual Theory of Choice under Risk. Econometrica, 55(1), 95-115. 